\title{
Fbxl17 is rearranged in breast cancer and loss of its activity leads to increased global 0 -GlcNAcylation
}

\author{
Bethany Mason ${ }^{1} \cdot$ Susanne Flach ${ }^{2,5} \cdot$ Felipe R. Teixeira $^{1,6} \cdot$ Raquel Manzano Garcia $^{3} \cdot$ Oscar M. Rueda ${ }^{3}$. \\ Jean E. Abraham ${ }^{3,4} \cdot$ Carlos Caldas $^{3,4} \cdot$ Paul A. W. Edwards ${ }^{2,3} \cdot$ Heike Laman $^{1} \mathbb{D}$
}

Received: 4 April 2019 / Revised: 5 September 2019 / Accepted: 16 September 2019 / Published online: 27 September 2019

(c) The Author(s) 2019

\begin{abstract}
In cancer, many genes are mutated by genome rearrangement, but our understanding of the functional consequences of this remains rudimentary. Here we report the F-box protein encoded by FBXL17 is disrupted in the region of the gene that encodes its substrate-binding leucine rich repeat (LRR) domain. Truncating Fbxl17 LRRs impaired its association with the other SCF holoenzyme subunits Skp1, Cull and Rbx1, and decreased ubiquitination activity. Loss of the LRRs also differentially affected Fbxl17 binding to its targets. Thus, genomic rearrangements in FBXL17 are likely to disrupt $\mathrm{SCF}^{\mathrm{Fbx} 117}$-regulated networks in cancer cells. To investigate the functional effect of these rearrangements, we performed a yeast two-hybrid screen to identify Fbxl17-interacting proteins. Among the 37 binding partners Uap1, an enzyme involved in $O$-GlcNAcylation of proteins was identified most frequently. We demonstrate that Fbx117 binds to UAP1 directly and inhibits its phosphorylation, which we propose regulates UAP1 activity. Knockdown of Fbxl17 expression elevated $O$-GlcNAcylation in breast cancer cells, arguing for a functional role for Fbxl17 in this metabolic pathway.
\end{abstract}

Keywords FBXL17 · Genome rearrangements $\cdot O$-GlcNAcylation $\cdot O$-GlcNAc $\cdot$ UAP1 $\cdot$ Ubiquitin $\cdot$ Phosphorylation · Breast cancer

\section{Introduction}

The genomes of most common epithelial cancers, such as breast cancer, are highly rearranged, but our knowledge of the rearrangements and the genes they target remains rudimentary [1]. A few common, large-scale rearrangements

Bethany Mason and Susanne Flach contributed equally to this work.

Electronic supplementary material The online version of this article (https://doi.org/10.1007/s00018-019-03306-y) contains supplementary material, which is available to authorized users.

Heike Laman

hl316@cam.ac.uk

1 Department of Pathology at Tennis Court Road, University of Cambridge, Cambridge CB2 1QP, UK

2 Hutchison-MRC Research Centre, Addenbrooke's Site, Hills Road, Cambridge CB2 0XZ, UK

3 Department of Oncology, Cancer Research UK Cambridge Institute and Cancer Centre, Li Ka Shing Centre, University of Cambridge, Cambridge CB2 ORE, UK have been known for some time, such as loss of the distal arm of $8 \mathrm{p}, 17 \mathrm{p}$ and $18 \mathrm{q}$ and the amplification of ERBB2 in breast cancer, but many more less-frequently occurring aberrations remain to be characterised and may be diagnostically or therapeutically important. For example, the EML4$A L K$ fusion occurs in only approximately $5 \%$ of non-small cell lung cancers and is a target for therapy [2]. Genome sequencing has focused on point mutations in exomes, with only a few results for structural mutations reported so far, for limited sets of tumours [3-8]. Array-CGH detects larger scale unbalanced rearrangements and is available for large panels of tumours [9]. If such breaks fall within genes, they

4 Cambridge Breast Unit, NIHR Cambridge Biomedical Research Centre and Cambridge Experimental Cancer Medicine Centre at Cambridge University Hospitals NHS Foundation Trust, Cambridge CB2 2QQ, UK

5 Present Address: Department of Otolaryngology and Head \& Neck Surgery, Hospital of the Ludwig-Maximilians-University, Munich, Germany

6 Present Address: Department of Genetics and Evolution, Federal University of São Carlos, São Carlos, São Paulo, Brazil 
A

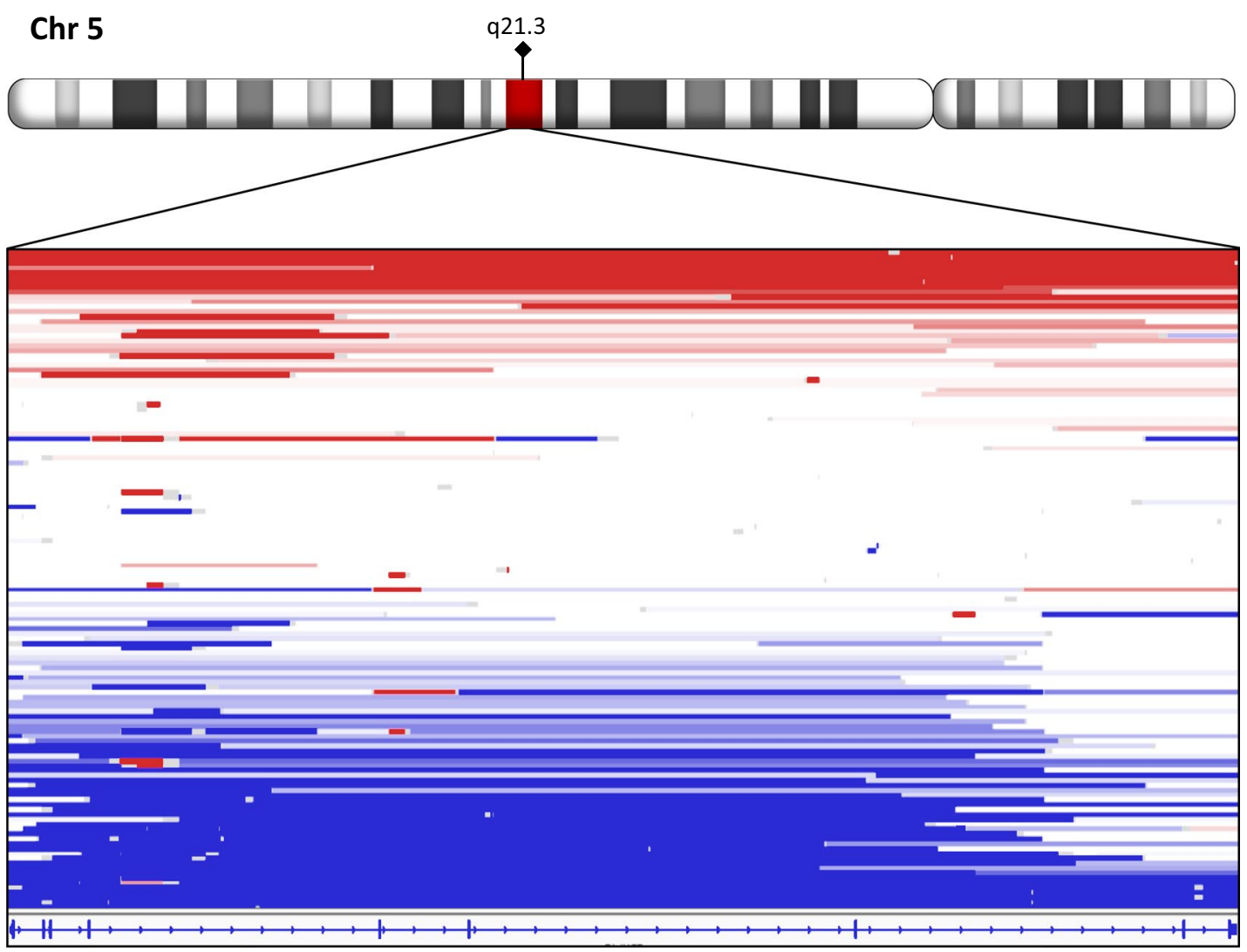

B
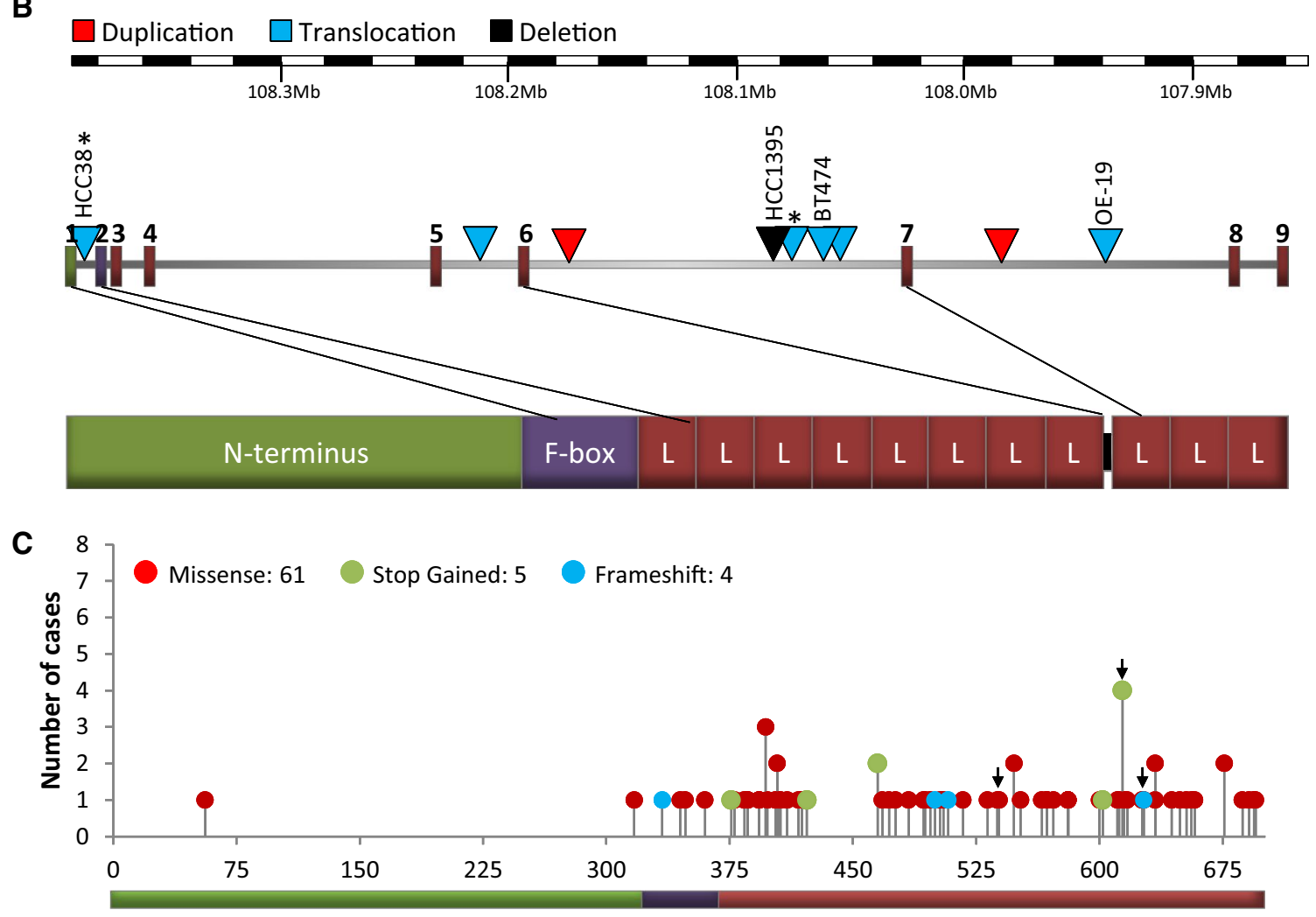

FBXL17 Transcript ENST00000542267 
4 Fig. 1 Breaks in FBXL17 and the proteins encoded. a Breaks in $135 / 1992$ breast tumours [9], detected as copy number steps by array-CGH. X-axis is genomic sequence of $F B X L 17$, reversed since $F B X L 17$ is a negative strand gene. Each horizontal line represents a tumour, with breaks indicated by colour change. Blue, copy number loss; white, no change; red, gain. b FBXL17 exons from Ensembl transcript ENST00000542267.5 (Refseq NM_001163315.2, NP_001156787.2), chr5:107,859,045-108,382,098 in GRCh38/hg38. Triangles indicate breakpoints in FBXL17 identified in cell lines (as labelled) or primary breast tumours (unlabelled), Asterisk indicates known fusion. Bottom, protein domains of Fbxl17 scaled to protein sequence. L, leucine-rich repeat. c Non-synonymous somatic mutations mapped to Fbx117 as reported by The Cancer Genome Atlas (TCGA), arrows indicate breast cancer associated mutations. Schematic underneath represents Fbxl17 domains, green, N-terminus, purple, F-box domain, red, leucine-rich repeats

must at least inactivate that copy of the gene, and in some cases they will create truncated proteins or gene fusions. Some gene fusion data are also available, from genomic [3-8] or transcript sequencing [10]. From surveying these datasets, we determined that FBXL17 is among the more frequently rearranged genes in a number of epithelial cancers, including breast, prostate and oesophageal cancers.

FBXL17 encodes a little-studied member of the F-box family of proteins (FBPs). They are components of the ubiquitin conjugation pathway, which, by directing the ubiquitination of target proteins, regulate major cellular processes that require rapid alterations in protein levels, activity and localisation, such as cell cycle progression, cell signalling, and receptor recycling [11]. Ubiquitination of proteins requires an enzymatic cascade involving an E1 ubiquitinactivating enzyme, an E2 ubiquitin-conjugating enzyme, and an E3 ubiquitin ligase [12]. FBPs are subunits of the SCF (Skp1-Cul1-F-box protein)-type E3 ubiquitin ligases, which utilise protein-protein interaction domains, like leucine-rich or WD40 repeats, to recruit substrates to the ligase. FBPs bind an adaptor protein Skp1 through their F-box domains (FBD), and the FBP:Skp1 dimer is a switchable unit that docks with a cullin scaffold and Rbx1 (Ring finger domain containing protein), which in turn recruits a ubiquitincharged E2 ligase. The Cand1 protein actively dissociates the pool of FBP:Skp1 dimers from cullin, regulating the levels of active E3 ligases in the cell [13, 14]. FBPs not engaged as part of active E3 ligases also have functions outside of the Ubiquitin Proteasome System (UPS) [15].

Several FBPs have oncogenic and/or tumour suppressive activities [16, 17]. Indeed, the first FBP described, Skp2 (S-phase kinase-associated protein 2, Fbxl1) is activated by amplification in several cancers, including breast, lymphoma, non-small cell lung cancer and glioblastoma [18, 19]. Skp2 is thought to have its main oncogenic activity by promoting the degradation of the cyclin-dependent kinase inhibitor, p27 [20-22]. However, loss of SKP2 also induces senescence in response to oncogenic stimuli, such as Ras expression or the loss of Pten [23]. Fbxw7 is a tumour suppressor, inactivated in approximately $7 \%$ cancers [24], which targets the turnover of important oncogenes, such as Myc, cyclin E, and Notch [16]. $\beta$-TrCP (BTRC, Fbxw11) is also mutated in several cancers, including breast and colorectal cancer and melanoma, potentially stabilising its oncogenic substrate $\beta$-catenin [19]. The true extent of FBP dysregulation in cancer, particularly through genomic rearrangements, is unknown. We found $F B X L 17$ is rearranged in breast cancers, and these rearrangements often disrupt the LRRs of Fbx117. Loss of LRRs leads to a differential loss of interaction with Fbxl17 binding partners, and prevents its assembly into a functional SCF complex. We show that Fbx117 interacts with Uap1, UDP- $N$-acetylglucosamine pyrophosphorylase 1 , to regulate the overall levels of $N$-acetylglucosamine modification ( $O$-GlcNAcylation) of proteins in cells. Our data support a model whereby Fbx117 has tumour suppressor activity in breast cancers.

\section{Results}

\section{FBXL17 is rearranged in cancer}

To identify genes that are rearranged in breast cancers, we scanned segmented array-CGH copy number data for 1992 primary breast tumours [9]. 135 (7\%) had at least one genomic break within $F B X L 17$, detected as a copy number step, distributed in various ways (Fig. 1a). The majority of these copy number losses or gains occurred at the $3^{\prime}$ end of FBXL17. Given the LRRs of Fbxl17 are encoded from exon 3 onwards it is likely these protein-protein interacting domains are disrupted by such rearrangements.

In addition to breaks in tumours, array-CGH data showed breaks in FBXL17 in four cancer cell lines, the breast carcinoma cell lines, BT-474, HCC38, and HCC1395, and the oesophageal/gastric cardia adenocarcinoma line OE-19 [25]. The breaks were verified by FISH (Figs. 1b and S1). In BT-474, one of three copies of FBXL17 was broken, with retention of the $3^{\prime}$ end, exons 7-9 (Figs. $1 \mathrm{~b}$ and S1B). Both HCC38 and OE-19 had an extra copy of the 5' end of FBXL17, up to intron 1 and intron 7, respectively (Figs. $1 \mathrm{~b}$ and $\mathrm{S} 1 \mathrm{~B})$. In HCC38, this break was confirmed to be the FBXL17-PJA2 fusion transcript reported in [10] (Fig. S1C, S1F and S1G). In HCC1395, both array-CGH (Fig. S1H) and paired-end sequencing showed an internal homozygous deletion in FBXL17 between exons 6 and 9 [5, 25] verified by RT-PCR and FISH (Fig. S1D, S1E and S1I) which would truncate Fbx117 near its C-terminus and encode a mutant protein lacking approximately three LRRs (Fig. 1b).

Because the cell line examples may not be typical, we looked for examples of FBXL17 rearrangements in breast cancers, in paired-end whole-genome DNA sequencing 
A

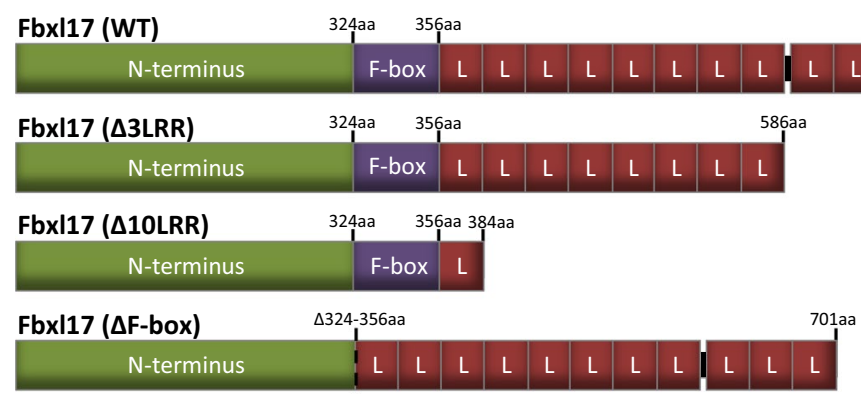

C

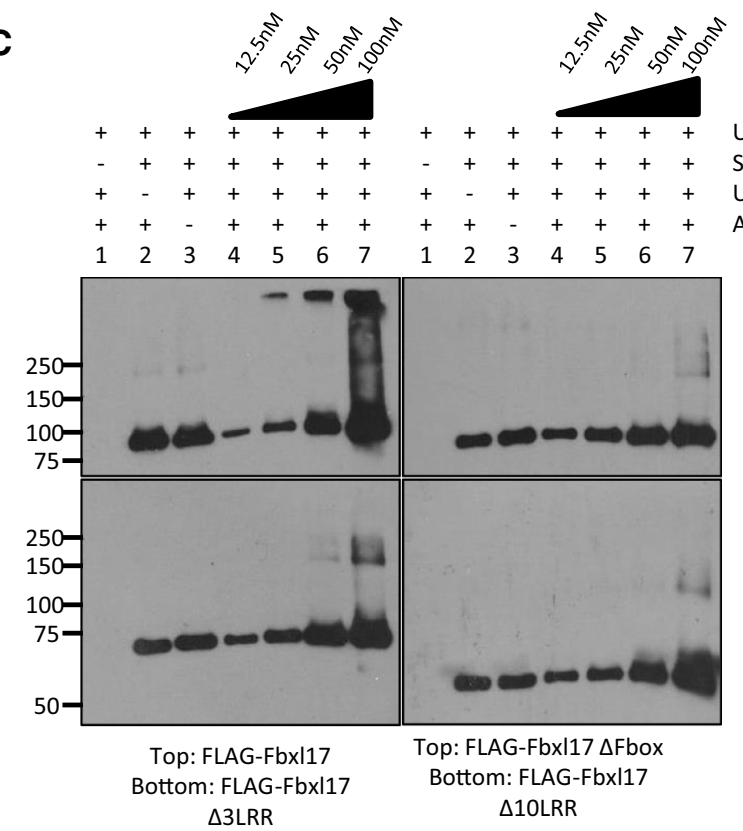

$\mathbf{E}$

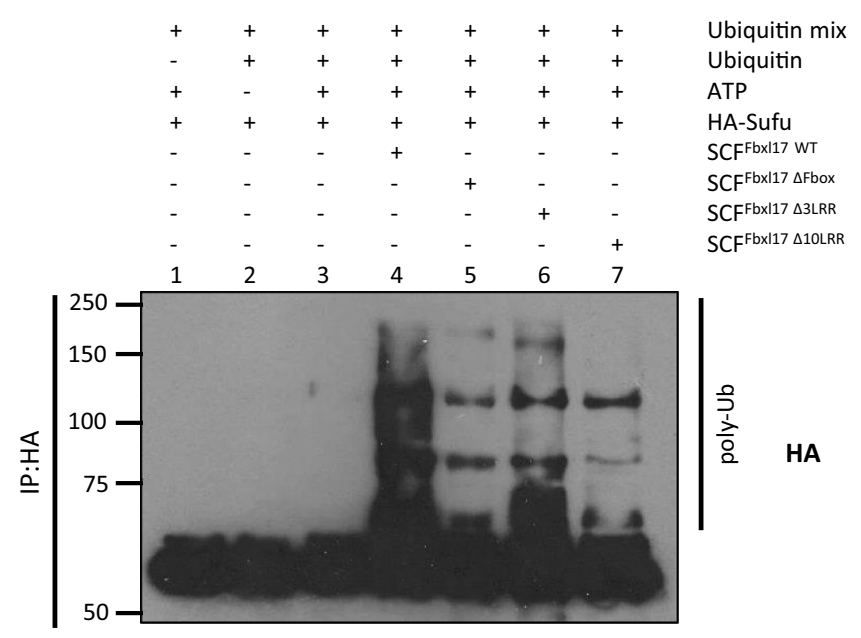

B $\quad+\quad+\quad+\quad+\quad$ Cul1, Skp1, myc-Rbx1

FLAG- Fbxl17 WT

FLAG- Fbxl17 $\triangle$ Fbox

FLAG-Fbxl17 $\triangle 3$ LRR

FLAG-FbxI17 $\triangle 10 \mathrm{LRR}$
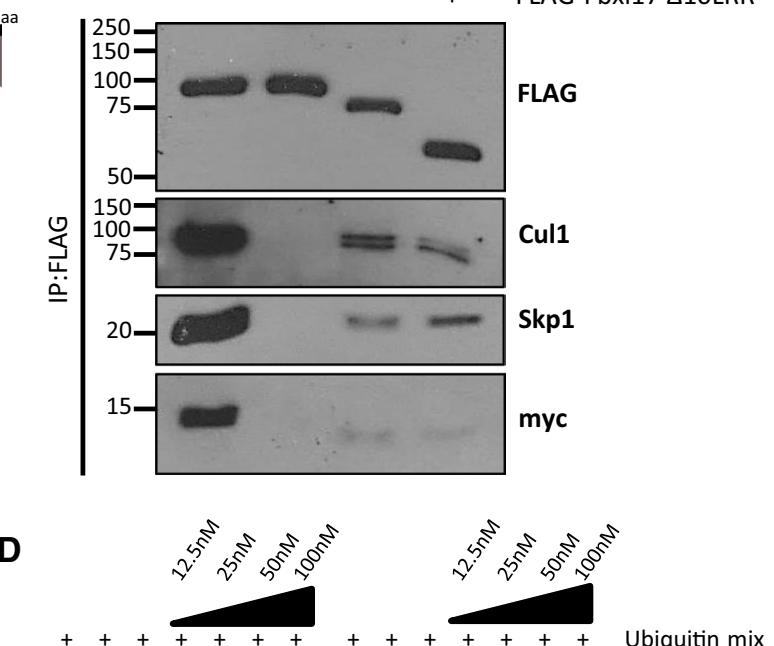

SCF complex $\quad-++++++-++++++$ SCF complex Ubiquitin +-++++++-+++++ Ubiquitin

ATP $\quad++-++++++-+++$ ATP

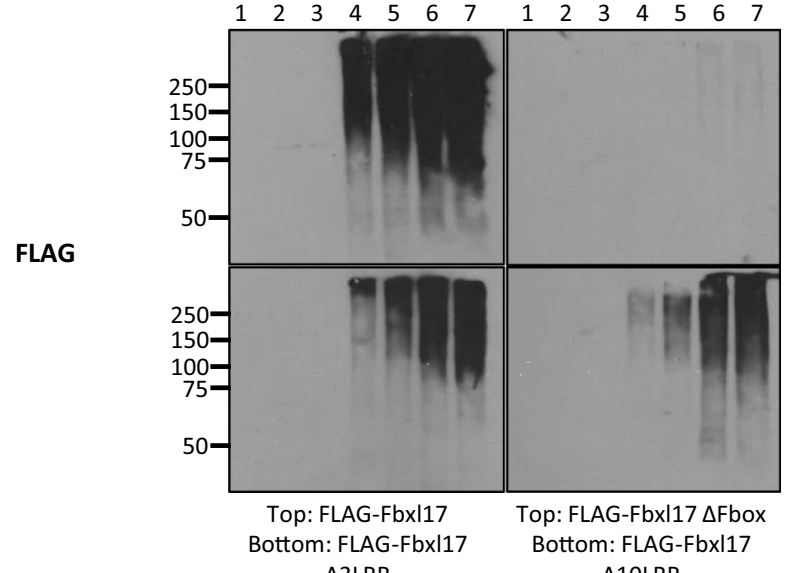
$\triangle 3$ LRR $\triangle 10 \mathrm{LRR}$ 
4Fig. 2 Loss of Fbx117 LRRs impairs recruitment of $\mathrm{SCF}^{\mathrm{Fbx} 117}$ subunits and $\mathrm{SCF}^{\mathrm{Fbxl17}}$ ligase activity. a Schematic showing Fbxl17 constructs used to make SCF ligases, all contain an N-terminal FLAG tag (not shown). L, leucine-rich repeat. b A representative immunoblot for SCF holoenzyme components that co-immunoprecipitate with FLAG-Fbx117 and mutant Fbx117 constructs FLAG-Fbx117 $\Delta$ Fbox, FLAG-Fbx117 $\Delta 3$ LRR and Fbx117 $\Delta 10 L R R, n=4$. $\mathbf{c}$ Titration of the auto-ubiquitination activity of purified $\mathrm{SCF}^{\mathrm{Fbx} 117}$ ligase complexes. A concentration gradient $(12.5 \mathrm{nM}, 25 \mathrm{nM}, 50 \mathrm{nM}, 100 \mathrm{nM})$ of purified $\mathrm{SCF}^{\mathrm{Fbx} 117}$ or mutant complexes $\mathrm{SCF}^{\mathrm{Fbxl17} \Delta \mathrm{Fbox}}, \mathrm{SCF}^{\mathrm{Fbx} 117 \Delta 3 \mathrm{LRR}}$ or $\mathrm{SCF}^{\mathrm{Fbx} 117 \Delta 10 \mathrm{LRR}}$ was used in an in vitro ubiquitination assay in the presence of a ubiquitin mix (ubiquitin buffer, UBE1, UbcH5a and ATP). Following SDS-PAGE membranes were probed with anti-FLAG antibody to detect $\mathrm{SCF}^{\mathrm{Fbxl17}}$ ligases, $n=2$. d As (C) but probed with anti-ubiquitin antibody, $n=2$. e In vitro ubiquitination assay of $\mathrm{SCF}^{\mathrm{Fbx} 117}$ and mutant ligase complexes in combination with HA-tagged substrate Sufu in the presence of a ubiquitin mix as in c. Proteins resolved by SDS-PAGE and membrane probed with anti-HA antibody, $n=3$

data from 250 primary breast tumours of the Cambridge Personalised Breast Cancer Programme. Rearrangements ('Structural variants') in FBXL17 were identified in five of the tumours. Manual inspection of RNA sequences from these five tumours confirmed that two of the rearrangements were transcribed as predicted: a translocation joining exon 6 to an undocumented exon on chromosome 7 and a duplication of exon 6, respectively (Fig. 1b; Supplementary Table 1). A third case with a breakpoint in intron 6 showed unspliced transcription from exon 6 into intron 7; however, we cannot rule out that this was normal unspliced RNA. Serendipitously, a further RNA sample, inspected because it had a rearrangement which did not pass filtering, showed splicing from exon 6 into exon 4 . This suggested the presence of a rearrangement which was not detected by DNA sequencing in an additional tumour. The partial agreement we find between RNA and DNA sequencing is expected as both methods lack sensitivity to identify all rearrangements [26]. Thus, consistent with the cell line rearrangements, the breakpoints in the tumours fell within introns that would disrupt the expression of LRRs, with the majority (4/6) occurring in intron 6 (Fig. 1b, Supplementary Table 1). There was no clear relationship between FBXL17 rearrangement and any molecular classification [27] of the tumours and cell lines, although five of the six tumours and the breast cell lines were TP53 mutant (Supplementary Table 1). Of the tumours, three were oestrogen receptor (ER) positive, and three were ER-negative. One tumour and one cell line were ERBB2/HER2 positive. We also classified these tumours into the 11 IntClust sets [27], and they fell into three sets: clusters 4, 7 and 10. In addition, classifying these six cases using PAM50 breast cancer subtyping, gave four basal and two luminal A cases. Although the number of cases is small, these data suggest Fbxl17 is not rearranged in a particular cancer subtype.
Rearrangements of $F B X L 17$ have also been detected in other epithelial cancers (Supplementary Table 2) including prostate [8] and oesophageal adenocarcinoma [7]. Many of these rearrangements are also predicted to truncate Fbx117, resulting in loss of LRRs. TCGA (Cancer Genome Atlas project) data was mined for genomic alterations affecting FBXL17 using cBioPortal (http://cbioportal.org) [28]. Perhaps most striking was the TCGA mapping of non-synonymous somatic FBXL17 mutations. Mutations in FBXL17 almost exclusively (68/70) target its C-terminus containing the FBD and LRRs (Fig. 1c).

In summary, FBXL17 is broken in approximately $7 \%$ of breast cancers, and additionally rearranged or mutated in other epithelial cancers. At least some of the breaks truncate Fbxl17, removing some or all of the LRRs and sometimes also the FBD. Examples of truncation are present in three cancer cell lines, and rearrangements have been confirmed in primary breast tumours. These genomic alterations suggest the ability of Fbxl17 to recruit substrates for ubiquitination or to form part of an SCF complex may be compromised.

\section{Deletion of LRRs in Fbxl17 compromises ubiquitination activity due to impaired recruitment of SCF subunits}

As most of the genomic rearrangements in FBXL17 are predicted to target its LRRs, we wanted to investigate the effect of their loss on Fbxl17 ligase activity. We used coimmunoprecipitation assays to check the incorporation of Fbx117 into an SCF E3 ligase. HEK293T cells were co-transfected with the subunits of SCF ligases, Skp1, Cullin1, Rbx1, and various N-terminally FLAG-tagged Fbx117 constructs (full-length Fbxl17 (1-701aa), an internal FBD deletion, Fbx117 $\Delta$ Fbox $(\Delta 324-358$ aa) and two LRR-truncation constructs Fbx117 $\triangle 3$ LRR (1-586aa), and Fbxl17 1 10LRR (1-384aa) (Fig. 2a). 48 h post transfection, cells were lysed, immunoprecipitated with FLAG antibodies, and blotted for the associated SCF subunits (Figs. 2b and S2B). While Skp1, Rbx1 and Cullin1 coimmunoprecipitated efficiently with WT Fbxl17, these components were reduced in the immunoprecipitates of the truncation mutants. For example, Cullin1 binding to Fbx117 $\Delta$ LRR was reduced by $81 \%(p=4.12 \mathrm{E}-04 ; \mathrm{n}=4)$ and Fbx117 $\Delta 10 \mathrm{LRR}$ by $82 \%(p=1.28 \mathrm{E}-06 ; n=4)$ relative to WT Fbx117, while Skp1 binding to Fbx117 $\Delta$ LRR was reduced by $77 \%(p=5.52 \mathrm{E}-05 ; n=5)$ and Fbxl17 $\Delta 10 \mathrm{LRR}$ by $67 \%(p=2.74 \mathrm{E}-05 ; n=4)$ relative to WT Fbxl17, despite these truncations having intact FBDs (Figs. 2b and S2B). As expected, when the FBD was deleted in Fbxl17 $\Delta$ Fbox, none of the subunits were co-immunoprecipitated. These data indicate that in addition to the FBD, the LRRs of Fbx117 facilitate the assembly of the $\mathrm{SCF}^{\mathrm{Fbx} 117}$ ligase. 
Since the Cullin1 and Rbx1 subunit allow E2 recruitment, these data suggest that the mutant $\mathrm{SCF}^{\mathrm{Fbx} 117}$ ligases will have reduced activity. We tested this by performing in vitro ubiquitination assays of purified SCF complexes, assembled with either WT or LRR-truncated Fbxl17 proteins, in the presence of an E1 and E2 enzyme. We first tested the ability of the mutant Fbxl17 proteins to promote auto-ubiquitination as part of an SCF E3 ligase (Figs. 2c, d and S2A). We observed higher molecular weight bands using an antibody raised against Fbx117 using $25 \mathrm{nM}$ of $\mathrm{SCF}^{\mathrm{Fbx} 117}$ in in vitro ubiquitination reactions, and the signal intensified with increasing concentrations of the WT ligase. In parallel assays, $50 \mathrm{nM}$ of mutant $\mathrm{SCF}^{\mathrm{Fbx} 117 \triangle 3 \mathrm{LRR}}$ ligase showed residual activity which increased at $100 \mathrm{nM}$, but it was considerably less than WT $\mathrm{SCF}^{\mathrm{Fbx} 117}$ ligase. $\mathrm{SCF}^{\mathrm{Fbx} 117 \Delta 10 \mathrm{LRR}}$ ligase showed the greatest reduction in activity (Fig. 2c), comparable to the inactive $\mathrm{SCF}^{\mathrm{Fbx} 117 \Delta \mathrm{Fbox}}$ mutant. The difference in activity of the SCF complexes was even more apparent when the membranes were probed for ubiquitin (Fig. 2d). SCF ${ }^{\mathrm{Fbx} 117 \Delta \mathrm{Fbox}}$ had no ligase activity, while E3 ubiquitin ligases made with the LRR-truncated Fbx117 mutants had reduced ligase activity compared to WT Fbxl17.

To test whether the ligases made by WT or mutant versions of Fbxl17 could ubiquitinate a heterologous substrate, we performed in vitro ubiquitination assays using Sufu (Fig. 2e) [29]. HA-Sufu was purified from HEK293T cells by immunoprecipitation. High molecular weight smears can be seen after the addition of $\mathrm{SCF}^{\mathrm{Fbx} 117}$ (Fig. 2e, lane 4) and to a much lesser extent $\mathrm{SCF}^{\mathrm{Fbx} 117 \Delta 3 \mathrm{LRR}}$ (Fig. 2e, lane 6). Both $\mathrm{SCF}^{\mathrm{Fbx} 117 \Delta \mathrm{Fbox}}$ and $\mathrm{SCF}^{\mathrm{Fbx} 117 \Delta 10 \mathrm{LRR}}$ show greatly reduced ubiquitination activity. Together these data indicate that the LRRs in Fbxl17 contribute to assembly of the SCF E3 ligase and its ligase activity.

\section{Fbxl17 interacting proteins identified by yeast-two hybrid screening}

Our data suggest that if Fbxl17 is mutated in the LRRencoding region, the proteins interacting with them will be mis-regulated as a result of aberrant SCF assembly and its effects on ligase activity. We performed a yeast two-hybrid screen to identify Fbx117 interacting partners. To focus the screen on LRR-binding partners, we engineered the bait plasmid to contain the FBD and LRRs (321-701aa) of Fbxl17 but omitted its N-terminus. 37 unique prey, cloned in-frame to the Gal4 activation domain (GAD), were identified as candidate partners for Fbxl17 (Table 1; Fig. S3A). More than a third (13/37) of the prey were isolated independently at least twice. The most common prey plasmids isolated encoded GAD fusions to UDP- $N$-acetylglucosamine pyrophosphorylase 1 (Uap1) and to ubiquitin-fold modifier conjugating enzyme 1 (Ufc1), which were isolated 26 and 13 times, respectively. Moreover, since multiple, non-identical plasmids were isolated, a minimal common region defined a likely interacting domain within it, e.g. aa 357-505 at the C-terminus of Uap1 (Table 1). Additionally, Klhl12 and Klhl7, two members of the Kelch-like family of proteins, casein kinase 2b (Csnk2B), rearranged L-Myc fusion (Rlf), and C21 orf91 were repeatedly isolated. To test whether the interaction between Fbxl17 and its prey was dependent on its LRRs, three LRRs ( $\triangle 3$ LRR) were deleted from the bait plasmid. Although this truncation did not affect Fbxl17 expression (Fig. S3B), none of the yeast co-transformed with the $\Delta 3$ LRR bait plasmid and the various prey grew under the selective conditions requiring a bait-prey interaction. These results indicated Fbxl17 interaction with its prey was dependent on its three C-terminal LRRs (Fig. S3A).

\section{Fbxl17 interacts with Uap1, Ufc1, KIhI12 and Csnk2B in human cells in vivo}

To validate the yeast two-hybrid results, we tested Uap1, Ufc1, Csnk2B and Klhl12 for their interaction with Fbx117 in human cells, using co-immunoprecipitation assays. All four proteins tested were detected in immunoprecipitates of FLAG-tagged Fbxl17 (Fig. 3a-d). We noted truncation of LRRs resulted in increased expression of mutant Fbxl17 (Fig. 2b-d). Despite their enhanced expression, Klhl12 did not interact with either the $\triangle 3$ LRR or $\Delta 10 \mathrm{LRR}$ mutants, indicating its interaction with Fbxl17 was dependent on the LRRs (Fig. 3a). Uap1 and Ufc1 both co-immunoprecipitated with WT and $\triangle 3$ LRR Fbx117, but truncation of 10 LRRs ablated their interaction (Fig. 3b, c). Uap1 was also present in immunoprecipitates of the mutant FLAG-Fbxl17 $\Delta$ Fbox indicating that Uap1 binding is dependent on LRR2-8 in Fbxl17 (Fig. S3C). In contrast to the other partner proteins, HA-Csnk2B co-immunoprecipitated roughly equivalently with WT, $\triangle 3 \mathrm{LRR}$, and $\triangle 10 \mathrm{LRR}$ constructs, suggesting that their interaction does not rely on the LRRs and thus may interact via the FBD or N-terminus of Fbxl17 in human cells (Fig. 3d).

The diminished interactions of truncated Fbxl17 with some of its binding partners could be caused by a change in its subcellular distribution. Cellular fractionation and immunofluorescence assays were conducted in parallel to determine the distribution of the WT and mutant proteins in cells. Endogenous Fbx117 was present in both cytoplasmic and nuclear fractions (Figs. S3D, S4A). Immunofluorescence showed all transfected Fbxl17 constructs showed a predominantly nuclear localisation and also weaker cytoplasmic staining (Fig. S4A). This argues against an altered localisation preventing the mutant forms from interacting with its binding partners.

In sum, these data validate results from the yeast twohybrid screen since the binding partners identified also interact in human cells with the full length Fbxl17 protein. 
Table 1 Fbxl17 interacting proteins

\begin{tabular}{|c|c|c|c|c|c|}
\hline Gene symbol & Full name & isolates & Minimal region (aa) & $\begin{array}{l}\text { Full-length } \\
\text { size (aa) }\end{array}$ & Modified \\
\hline UAP1 & UDP- $N$-acetylglucosamine pyrophosphorylase 1 & 26 & $357-505$ & 505 & $\mathrm{Ub}$ \\
\hline$U F C 1$ & Ubiquitin-fold modifier conjugating enzyme 1 & 13 & $12-95$ & 167 & $\mathrm{Ub}$ \\
\hline$C S N K 2 B$ & Casein kinase 2 , beta polypeptide & 4 & $1-123$ & 215 & $\mathrm{Ub}$ \\
\hline KLHL12 & Kelch-like 12 (Drosophila) & 4 & $1-162$ & 568 & \\
\hline$R L F$ & Rearranged L-myc fusion & 4 & $1670-1914$ & 1914 & \\
\hline C21orf91 & EURL/Chromosome 21 open reading frame 91 & 4 & $1-178$ & 296 & \\
\hline$A C S B G 2$ & Acyl-CoA synthetase bubblegum family member 2 & 3 & $528-666$ & 666 & \\
\hline ETFA & Electron-transfer-flavoprotein, alpha polypeptide & 3 & $103-284$ & 333 & $\mathrm{Ub}$ \\
\hline METAP2 & Methionyl aminopeptidase 2 & 3 & $323-342$ & 478 & $\mathrm{Ub}$ \\
\hline$A K D 1$ & Adenylate kinase domain containing 1 & 2 & $415-624$ & 1911 & \\
\hline SCG5 & Secretogranin V (7B2 protein) & 2 & $1-188$ & 212 & \\
\hline TASP1 & Taspase, threonine aspartase, 1 & 2 & $135-318$ & 420 & \\
\hline KLHL7 & Kelch-like 7 (Drosophila) & 2 & $37-240$ & 586 & $\mathrm{Ub}$ \\
\hline PHF7 & PHD finger protein 7 & 1 & $50-236$ & 381 & \\
\hline ZMYM2 & Zinc finger, MYM-type 2 & 1 & $141-390$ & 1377 & \\
\hline IDOI & Indoleamine 2,3-dioxygenase 1 & 1 & $14-128$ & 403 & \\
\hline PSME4 & Proteasome (prosome, macropain) activator subunit 4 & 1 & $367-420$ & 1843 & $\mathrm{Ub}$ \\
\hline$P P P 3 C B$ & Protein phosphatase 3 , catalytic subunit, beta isozyme & 1 & $381-496$ & 524 & $\mathrm{Ub}$ \\
\hline ZNF350 & Zinc finger protein 350 & 1 & $310-532$ & 532 & \\
\hline$T G F B I$ & Transforming growth factor, beta-induced, $68 \mathrm{kDa}$ & 1 & $115-326$ & 683 & \\
\hline SCPEP1 & Serine carboxypeptidase 1 & 1 & $273-452$ & 452 & $\mathrm{Ub}$ \\
\hline FILIP1L & Filamin A interacting protein 1-like & 1 & $508-705$ & 1135 & \\
\hline$A C P L 2$ & Acid phosphatase-like 2 & 1 & $266-464$ & 480 & \\
\hline$T P P 2$ & Tripeptidyl peptidase II & 1 & $879-1141$ & 1249 & $\mathrm{Ub}$ \\
\hline OLR1 & Oxidized low density lipoprotein (lectin-like) receptor 1 & 1 & $124-273$ & 273 & \\
\hline USP25 & Ubiquitin specific peptidase 25 & 1 & $323-450$ & 1055 & $\mathrm{Ub}$ \\
\hline FAM190A & Family with sequence similarity 190 , member A & 1 & $555-726$ & 900 & \\
\hline$P C C B$ & Propionyl CoA carboxylase, beta polypeptide & 1 & $172-468$ & 539 & \\
\hline TMOD1 & Tropomodulin 1 & 1 & $66-334$ & 359 & \\
\hline IFT46 & Intraflagellar transport 46 homolog (Chlamydomonas) & 1 & $1-216$ & 304 & \\
\hline$S R B D 1$ & S1 RNA binding domain 1 & 1 & $260-491$ & 995 & \\
\hline$M E D 14$ & Mediator complex subunit 14 & 1 & $1053-1282$ & 1454 & $\mathrm{Ub}$ \\
\hline CCDC147 & Coiled-coil domain containing 147 & 1 & $478-684$ & 872 & \\
\hline$H A D H$ & Hydroxyacyl-CoA dehydrogenase & 1 & $112-261$ & 261 & $\mathrm{Ub}$ \\
\hline$C L P X$ & ClpX caseinolytic peptidase $\times$ homolog (E. coli) & 1 & $42-341$ & 633 & \\
\hline COG2 & Component of oligomeric golgi complex 2 & 1 & $457-534$ & 738 & $\mathrm{Ub}$ \\
\hline TMEM126A & Transmembrane protein $126 \mathrm{~A}$ & 1 & 89-195 & 195 & $\mathrm{Ub}$ \\
\hline
\end{tabular}

Minimal region denotes the amino acids present in all interacting cDNA clones; full-length size is the predicted size of the protein. Ub denotes protein is ubiquitinated in [39]

Furthermore, the association of Klhl12, Ufc1, and Uap1 with Fbxl17 was dependent on its LRRs, as truncation of this region weakened or ablated their interaction.

To test the directness of the interaction between Fbx117 and one of its interacting proteins, we performed a GST pull-down assay using Uap1. We tested GST-Fbxl17(321701aa), GST-Fbxl17 10 LRR(321-383aa), and GST-Skp2, another LRR-containing FBP, and GST only were used as controls. GST-FBP proteins were co-expressed with an IRES-Skp1 to facilitate expression in bacteria, with the exception of Fbx117 $10 \mathrm{LRR}$, which was robustly expressed. GST-FBPs were immobilised on a GST column and incubated with in vitro transcribed and translated Uap1. Following binding assays, samples were resolved by SDS-PAGE, and membranes probed with antibodies 

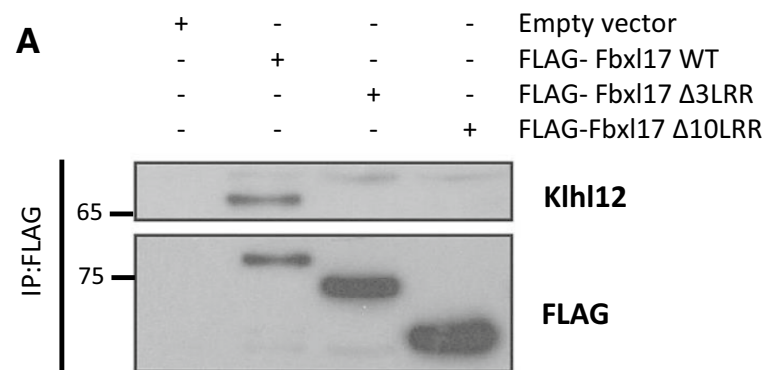

KIhl12

FLAG

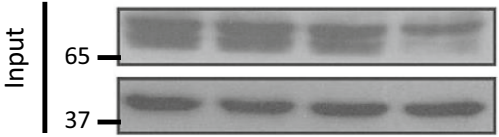

KIhl12

$\beta$-actin

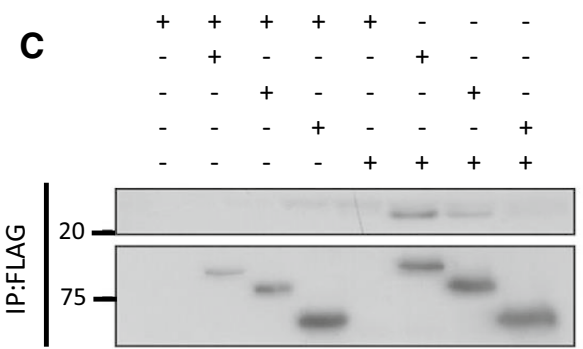

Empty vector

FLAG- Fbxl17 WT

FLAG- Fbxl17 $\triangle 3$ LRR

FLAG-Fbxl17 $\triangle 10 L R R$

HA-Ufc1

HA

FLAG

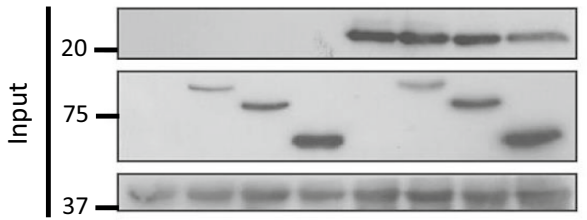

HA

FLAG

$\beta$-actin

Fig. 3 Deletion of the LRRs of Fbx117 affects its binding to different proteins to different extents. a Immunoprecipitates; using antiFLAG beads, of HEK293T cells expressing FLAG-Fbx117, FLAG-

to Uap1. We observed Uap1 binding specifically to GSTFbx117(321-701aa), but not to GST only or to GST-Skp2. Moreover, deletion of 10 LRRs abolished Uap1 binding to Fbxl17 (Fig. 4a). These results indicate Fbxl17 interacts directly with Uap1, and this occurs via its LRRs.

\section{Fbxl17 inhibits the phosphorylation of Uap1}

To test the functional significance of Fbx117 interaction with Uap1, we over-expressed Fbx117 and monitored the steady state levels of Uap1 by immunoblotting. We found Uap1 levels were unchanged in the presence of MG132 or with increased levels of Fbxl17 (Fig. 4b), which suggests it does not promote the proteasomal degradation of Uap1. We next tested whether Uap1 was a substrate of $\mathrm{SCF}^{\mathrm{Fbx} 117}$ ligase in vivo, by co-transfecting cells with HA-Uap1, Myc-ubiquitin, and FLAG-Fbx117 (WT or $\Delta$ F-box domain) constructs. However, we found no evidence of laddering or smearing
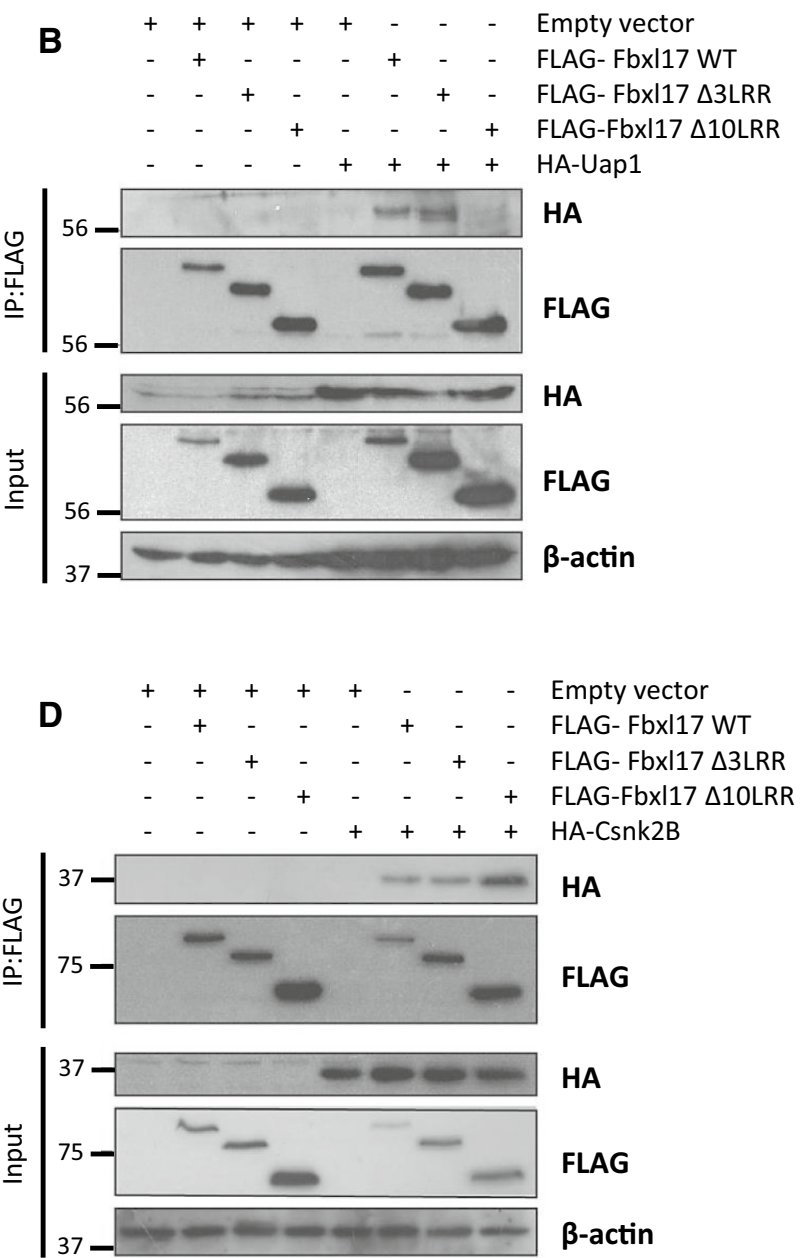

Fbx117 3 LRR or FLAG-Fbx117 10 LRR, probed for endogenous Klhl12. b-d As (a), but with co-expression of exogenous HA-tagged Uap1 (b), Ufc1 (c), or Csnk2B (d) and probed with anti-HA antibody

of Uap1, indicative of its poly-ubiquitination. Instead, we detected a discrete, higher molecular weight species of Uap1 upon transfection of Myc-ubiquitin (Fig. 4c, lane 2). Moreover, levels of this modified form of Uap1 were reduced when Fbxl17 was overexpressed (Fig. 4c, lane 5), indicating Fbxl17 opposed this modification of Uap1. Interestingly, this reduction of Uap1 modification was not observed when Fbxl17 $\Delta$ Fbox was overexpressed, (Fig. 4c, lane 6) suggesting this effect was dependent on Skp1 binding and/or the ligase activity of Fbxl17.

To determine the type of modification this higher molecular weight species of Uap1 represented, we immunoblotted with antibodies to ubiquitin and to Myc-epitope tag (Mycubiquitin). Surprisingly, these antibodies did not yield any signal in Uap1 immunoprecipitates, despite the overexpression of ubiquitin (S2C and S2D). These results indicated that the post-translational modification present on Uap1 was not ubiquitination. Based on the PhosphoSitePlus database, 
A
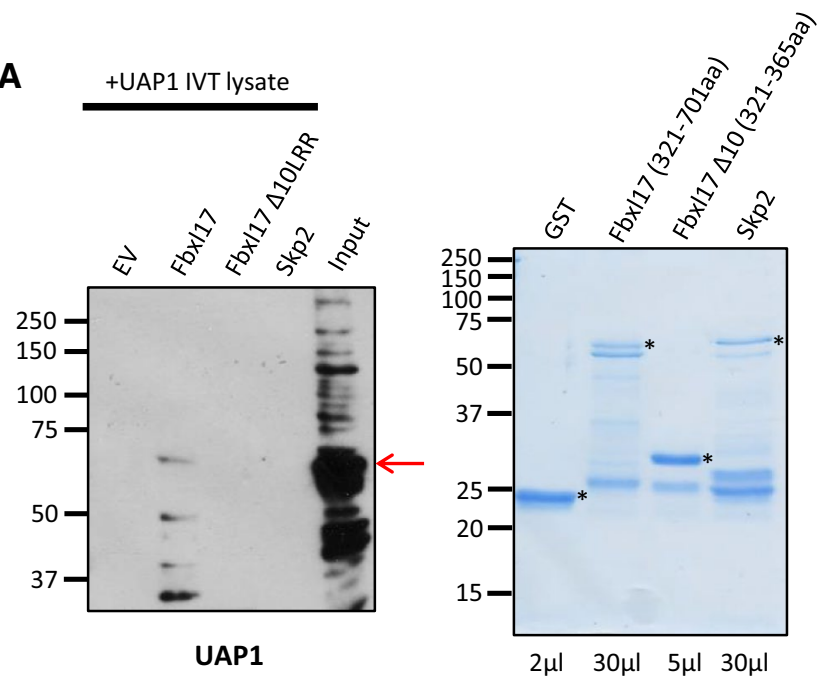

C

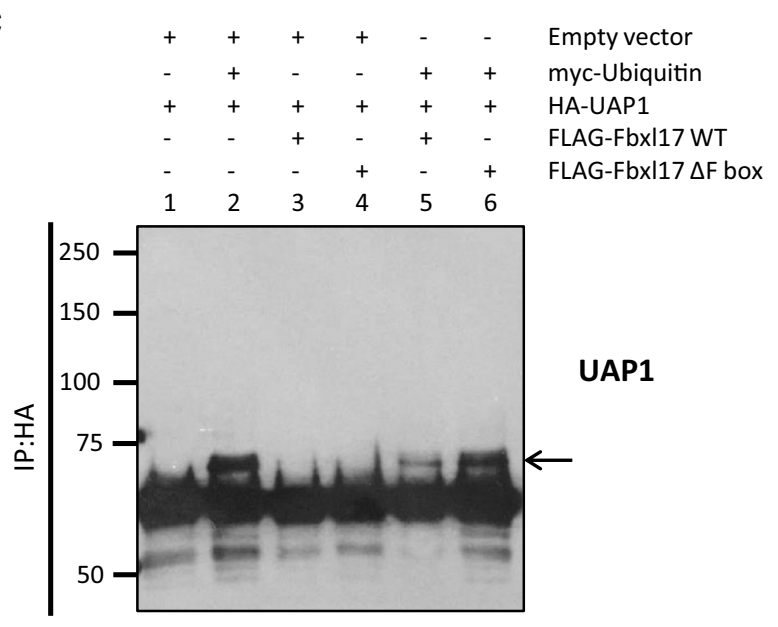

E UDP-GICNAC

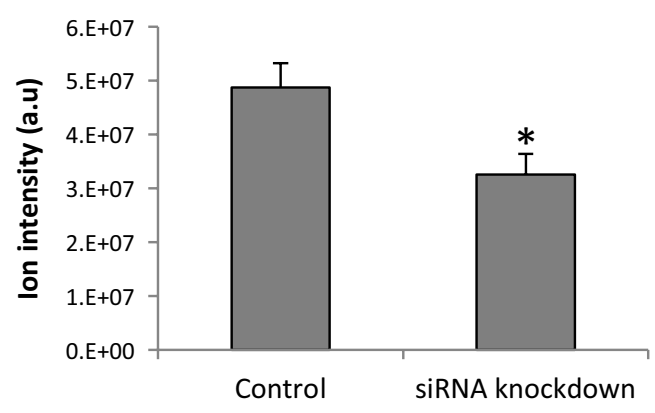

Fig. 4 Fbx117 inhibits the phosphorylation of UAP1. a In vitro GST pull-down assay using bacterially expressed and purified Fbx117 constructs or Skp2/GST as controls immobilised on a GST column incubated with rabbit reticulocyte lysate (left panel). Fbxl17(321-701aa) and Skp2 constructs contained an IRES_Skp1 to aid expression. Input for rabbit reticulocyte lysate $=20 \%$. Coomassie staining of GST proteins, volume of sample loaded indicated below lanes (right panel) * indicates bands relating to expressed proteins, arrow represents Uap1, $n=2$. b HEK293T cells transfected with Fbxl17WT, $\Delta$ Fbox or empty vector $(\mathrm{EV})$ for $48 \mathrm{~h}$ then treated with $10 \mu \mathrm{M}$ MG132 or DMSO for
B

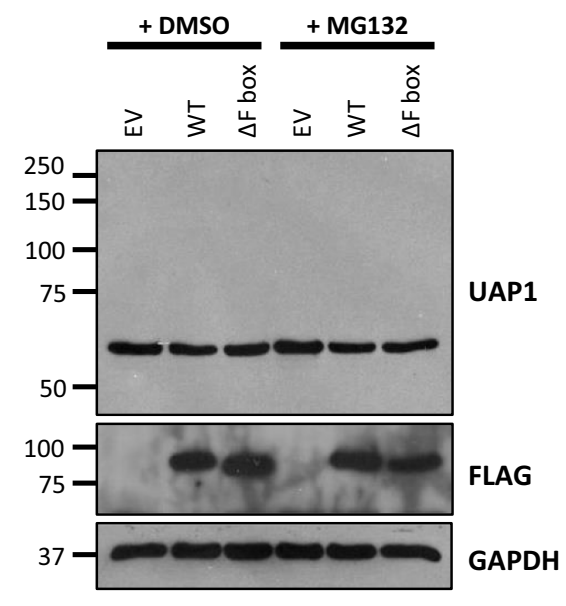

D

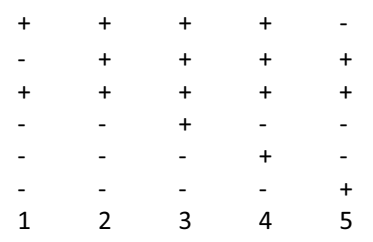

Empty vector myc-Ubiquitin HA-UAP1 $\beta$-glycerophosphate Cip phosphatase FLAG-Fbxl17 WT

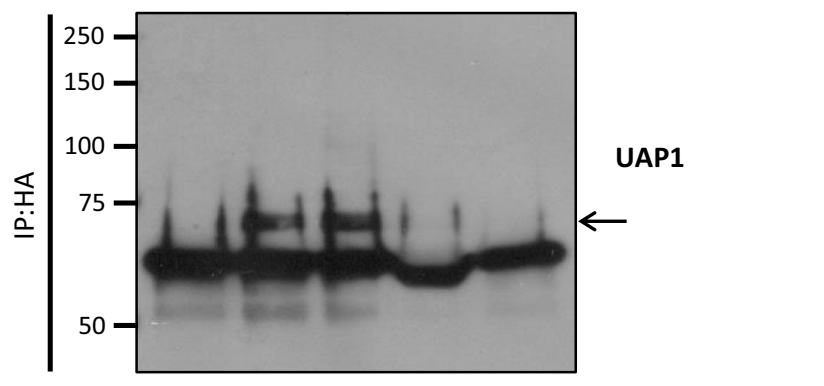

$4 \mathrm{~h}$. Whole cell lysates immunoblotted with the indicated antibodies, $n=3$. c In vivo ubiquitination assay for UAP1. HA-UAP1 immunoprecipitated from HEK293T cells transfected with ubiquitin and indicated Fbx117 constructs. Membranes probed with anti-UAP1 antibody, arrow indicates modified Uap1, $n=3$. d In vivo ubiquitination assay for UAP1 as in $\mathbf{c}$ in the presence of $\beta$-glycerophosphate (lane 3 ) and alkaline phosphatase (CIP) (lane 4), $n=2$. e LC-MS analysis of total UDP-GlcNAc levels in U2OS cells treated with Fbx117 siRNA3 or control siRNA for $48 \mathrm{~h}$. Mean \pm SEM for five biological replicates, $* p<0.05$ 
where multiple studies report Uap1 to be a phosphorylated protein, we tested whether this modified form of Uap1 represented a phosphorylated form. We conducted the in vivo ubiquitination assay in the presence of the phosphatase inhibitor $\beta$-glycerophosphate and alkaline phosphatase (CIP). Strikingly, the levels of modified Uap1 were almost completely absent following CIP treatment, suggesting the higher molecular weight species represented a phosphorylated form of Uap1 (Fig. 4d, lane 4). In sum, these data show that the overexpression of ubiquitin increases Uap1 phosphorylation, and Fbx117 overexpression prevents this modification, and this function is dependent on its FBD.

\section{FBXL17 knockdown results in increased levels of O-GIcNAcylation}

Since Fbx117 overexpression reduced the abundance of a phosphorylated Uap1, but not Uap1 steady state levels, we reasoned Fbx117 might regulate Uap1 activity. Uap1 catalyses the formation of UDP- $N$-acetylglucosamine (UDP-GlcNAc), which is used by the glycosyltransferase $O$-GlcNAc Transferase (OGT) to add $\mathrm{N}$-acetylglucosamine in $O$-glycosidic linkages to nuclear and cytosolic proteins. UAP1 is the main enzyme synthesizing UDP-GlcNAc [30-34]. Since our results indicate Fbx117 opposes Uap1 phosphorylation, we tested whether reducing Fbx117 expression would affect the amount of UDP-GlcNAc in the cell. U2OS cells were transfected with an siRNA targeting Fbxl17 (Fig. S4B), metabolites were extracted from these cells and UDP-GlcNAc levels were determined by mass spectrometry. We observed a $36 \%$ decrease $(p=0.013 ; n=5)$ in total UDP-GlcNAc levels in Fbxl17 knockdown cells (Fig. 4e), which suggests that Fbx117 promotes Uap1 activity.

To determine the downstream effects of Fbxl17 on this pathway, we next tested the effect of reduced Fbxl17 expression on the levels of $O$-GlcNAc modified cellular proteins. U2OS cells were treated with Fbxl17 siRNA as above, and cell lysates were immunoblotted for $O$-GlcNAc. Although UDP-GlcNAc levels were reduced in Fbx117 KD cells (Fig. 4e), we observed increased levels of $O$-GlcNAcmodified proteins in Fbxl17 knockdown cells (Fig. 5a). This was also shown in two breast cell lines, HB4a and MCF7, by expression of shRNA constructs targeting FBXL17 expression (Figs. 5b, c; S4C). This increase in $O$-GlcNAc modified proteins, despite reduced levels of UDP-GlcNAc, may be a result of changes in the expression or activity of the enzymes responsible for adding or removing $O$-GlcNAc, namely Ogt and Oga, which act downstream of Uap1 and UDP-GlcNAc. We therefore determined whether Ogt and Oga were changed following knockdown of Fbx117. Although Ogt levels were unchanged, Oga levels were reduced which suggests the increased $O$-GlcNAcylated proteins is due to decreased Oga levels (Fig. 5d). Consistent with reduced Oga levels, mass spectrometry analysis of levels of GlcNAc, the product of Oga-mediated cleavage of $O$-GlcNAc modifications from proteins, were reduced by $18 \%$, although this was not significant, in cells treated with Fbxl17 siRNA (Fig. 5e). Finally, we surveyed the expression of Fbxl17 and Uap1 expression in breast cancer datasets using the R2 platform (http://www. r2.amc.nl). Kaplan-Meier analysis for these genes revealed that breast tumours with either low Fbx117 expression, or high Uap1 expression were associated with poorer survival in patients (Fig. S5).

\section{Discussion}

Our results collectively indicate that $F B X L 17$ is frequently mutated in epithelial cancers in the genomic regions encoding its LRRs. We found FBXL17 was rearranged in around $7 \%$ of breast cancers according to array-CGH, and also in cancer cell lines. Independently, analysis of sequence-level mutation data also suggested that FBXL17 behaves like a tumour suppressor gene [35]. Many point mutations and breaks in FBXL17 occurred in regions encoding its LRRs. These motifs are proposed to be the substrate docking sites within FBXL proteins, and are predicted to cause a failure to recruit substrates. Indeed, we find that progressive deletion of its LRRs caused decreased auto-ubiquitination and ubiquitination of a substrate, Sufu, by $\mathrm{SCF}^{\mathrm{Fbx} 117}$. However, we also discovered that deleting LRRs impaired the assembly of the E3 ubiquitin-ligase complex. Both of the Fbxl17 LRR truncation mutants, $\triangle 3 \mathrm{LRR}$ and $\Delta 10 \mathrm{LRR}$, bound Skp1 less well compared to WT, despite them containing the Skp1 binding motif. Reduced interactions with Cul1 and Rbx 1 are likely due to less Skp1 binding, since FBPs do not interact directly with Cul1 or Rbx1 [36]. The C-terminus of an FBP has been shown in specific cases to contact Skp1 to stabilise the ligase [36], and our data show that truncating the LRRs of Fbxl17 also destabilises the SCF ligase. We predict that even if a truncated Fbxl17 were able to recruit some of its substrates, it would be less efficient in ubiquitinating them. Thus in the context of breast cancer, the rearrangements that target the LRRs of FBXL17 would likely diminish ubiquitination of the network of $\mathrm{SCF}^{\mathrm{Fbx} 117}$ substrates.

To investigate functional consequences of these rearrangements, we screened for proteins interacting with the LRR of Fbxl17. Our yeast two-hybrid screen identified 37 novel interactions, which was found to only minimally overlap with previous studies reporting Fbxl17 interacting proteins $[37,38]$. Only eight proteins, Klhl12, Klhl7, Zmym2, Hadh, Clpx, Ppp3cb, Pccb, Srbd1 also appeared in these other studies. However, Uap1 and Ufc1, the most frequently recovered cDNAs in our screen, were not identified by either. The varied findings by different screening methodologies, indicates they identify distinct partner proteins 
Fig. 5 Knockdown of Fbx117 increases total $O$-GlcNAcylation. a U2OS cells treated with Fbx117 siRNA3 or control siRNA for $48 \mathrm{~h}$ followed by PugNAc treatment, $50 \mu \mathrm{M} 3 \mathrm{~h}$. Whole cell lysates immunoblotted with the indicated antibodies, $n=2$. b $F B X L 17$ mRNA knockdown by shRNA in HB4a immortalised normal breast cells (bottom panel). Expression normalised to GAPDH and plotted relative to miR30-infected control cells. Mean \pm SEM of at least three independent experiments. $O$-GlcNAcylation monitored by immunoblotting with anti- $O$-GlcNAc antibodies (top panel). c FBXL17 mRNA knockdown by shRNA in MCF7 breast cancer cells (bottom panel). Expression normalised to scRNA-infected cells. Mean \pm SEM of at least three independent experiments. Total $O$-GlcNAcylation monitored by immunoblotting with anti$O$-GlcNAc antibodies. + and ,++ 25 or $50 \mu \mathrm{g}$ of protein lysate (top panel). d U2OS cells treated with Fbx117 siRNA3 or control siRNA for $48 \mathrm{~h}$. Whole cell lysates immunoblotted with the indicated antibodies. Band intensities quantified by densitometry and normalised to GAPDH expression (values below blots), $n=3$. e LC-MS analysis of total GlcNAc levels in U2OS cells treated with Fbx117 siRNA3 or control siRNA for $48 \mathrm{~h}$. Mean \pm SEM for five biological replicates. $\mathbf{f}$ Model of interaction between Fbx117 and Uap1

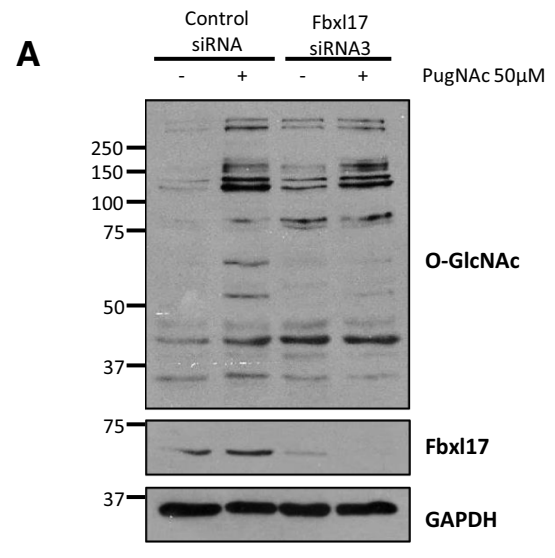

B
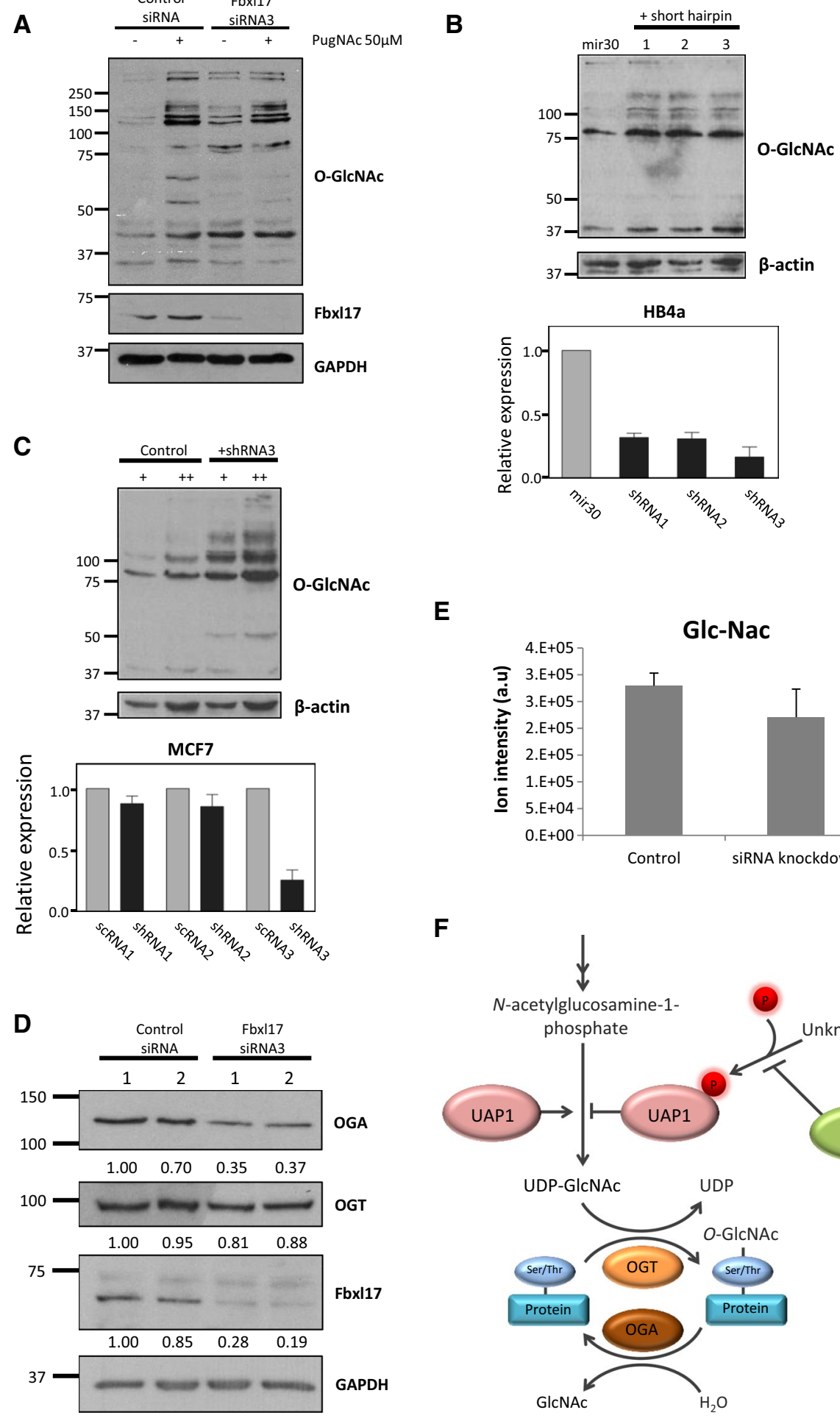

E

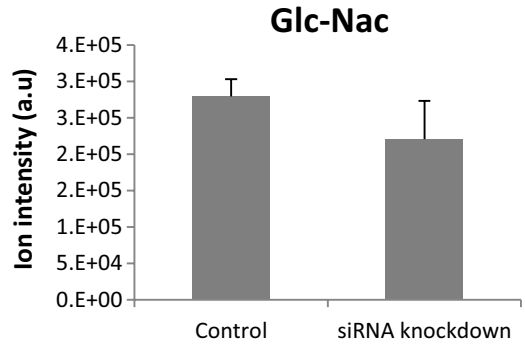

$\mathbf{F}$

O-GICNAC
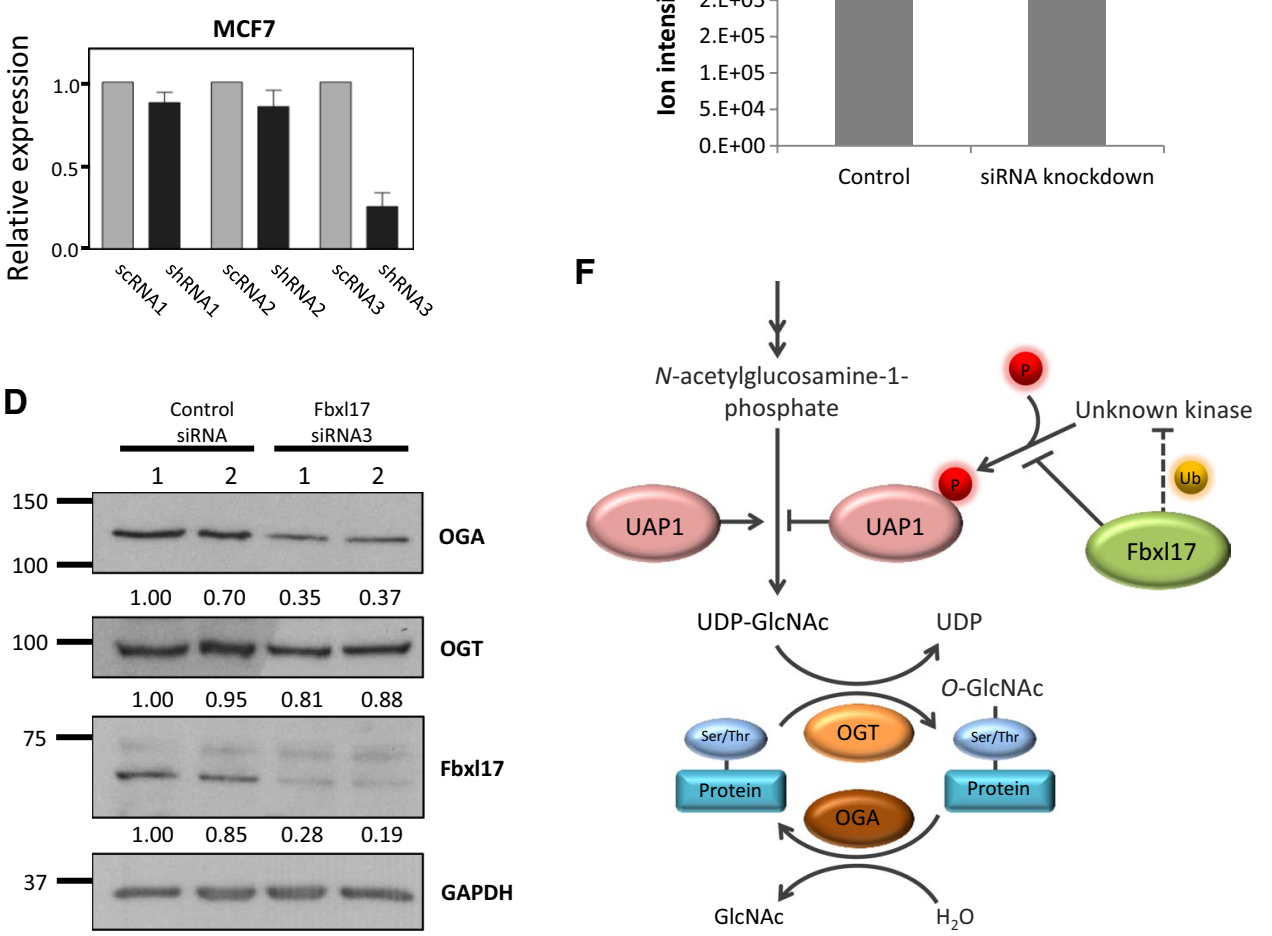

and argues for a variety of experimental approaches to discovering protein interaction networks. Screens for interacting partners may identify substrates or regulators of FBPs. When validating four of the most repeatedly isolated prey cDNAs in mammalian cells, we noted differential binding (Csnk2B $>$ Uap1 $>$ Ufc1 $>$ Klhl12) depending on the deletion of 3 or 10 LRRs. Notably, the Casein Kinase 2 subunit, Csnk2B, remained bound to Fbx117 after deletion of 10 LRRs, suggesting that it binds to another region of Fbxl17, or other E3 ubiquitin ligase components, and may be a regulator of the ligase. Among the other interactors, Fbxl17 binding to Klhl12 or Ufc1 was decreased when the last three 
LRRs were truncated. However, Uap1 binding was disrupted only when ten LRRs were truncated suggesting Uap1 can bind to LRR2-8. These data led us to a model whereby the number of LRRs in Fbxl17, as dictated by the position of a rearrangement within $F B X L 17$, would influence its interaction with its repertoire of partners. However, due to inefficient ligase assembly by LRR-truncated Fbx117 mutants, we predict the ubiquitination of these proteins would be diminished. We note that 15 of the 37 proteins identified in the yeast two-hybrid screen are listed as being ubiquitinated proteins in a whole proteomic analysis of HEK293 cells (Table 1) [39].

There are not yet enough data on rearrangement of $F B X L 17$ in cancers to conclusively identify FBXL17 inactivation as a driver mutation in cancer. Nonetheless, we were able to show a striking effect of reducing Fbxl17 expression on at least one important cancer-relevant pathway, in a relevant cell type, suggesting that inactivation of Fbxl17 would have a major effect on the cancer cell. This was through its regulation of Uap1, which is expressed in many breast cancers (Figs. S4D, S5D) and other cancer types [34, 40]. Surprisingly, we did not find Uap1 to be ubiquitinated by Fbxl17, but instead our results showed that increased Fbx117 expression prevented the phosphorylation of Uap1. In addition, this inhibition of Uap1 phosphorylation was dependent on the Skp1-binding domain of Fbxl17, suggesting that $\mathrm{SCF}^{\mathrm{Fbx} 117}$ ligase activity is essential. In contrast to Ogt and Oga [41], little is known about the regulation of Uap1, and our data suggest Fbxl17 positively regulates its activity (Fig. 5f). UDP-GlcNAc levels are significantly decreased when Fbxl17 is knocked down suggesting reduced Uap1 activity which is consistent with previous studies showing Uap1 expression is important for UDP-GlcNAc levels [34]. Although we have not identified the kinase responsible for phosphorylating Uap1, one possibility is that Fbx117 ubiquitinates this kinase to inhibit its phosphorylation of Uap1 and promote Uap1 activity. Alternatively, Fbxl17 could shield Uap1 from this kinase, via a direct interaction between Fbxl17 and Uap1 (Figs. 4a, 5f).

We have shown that Fbxl17 regulates the $O$-GlcNAcylation pathway since reducing $F B X L 17$ expression in three cell lines increased global levels of $O$-GlcNAc-modified proteins. We did not assess glycosylation in the ER or Golgi, so cannot rule out a specific role for Fbxl17 there. However, the increase in global $O$-GlcNAcylation may be the result of greater utilisation of UDP-GlcNAc by Ogt, which would explain the lower UDP-GlcNAc levels and higher $O$-GlcNAcylation we observed in Fbxl17 knockdown cells. Although Ogt expression levels were unchanged, we cannot rule out that its activity is increased when Fbxl17 levels are reduced. However, the higher levels of $O$-GlcNAc modified proteins are likely due to the observed decrease in Oga expression. It has been proposed that there is an optimal level of global $O$-GlcNAcylation levels for cells to function and this is maintained by mutual regulation and balance of Ogt/Oga expression and activity [41, 42]. The decrease in Oga expression may represent a compensatory mechanism adopted by the cell to counteract the decrease in Uap1 activity and UDP-GlcNAc levels. $O$-GlcNAcylation is an important post-translational modification on many intracellular proteins-including p53, RNA polymerase II, the polycomb complex and Phosphofructokinase 1 (Pfk1), the main regulator of glycolysis-and is essential for viability of several mammalian cell types [43, 44]. Moreover, there is already considerable evidence that GlcNAcylation is altered in breast cancer and other cancers [45-48]. Caldwell et al. [49] found that breast cancer cells had increased $O$-GlcNAcylation and elevated OGT. Knocking down $O G T$ inhibited tumour growth, decreased cell cycle progression, increased expression of the cell cycle inhibitor p27Kip1, and decreased invasiveness [49]. High nuclear and cytoplasmic O-GlcNAc was also observed in breast cancer patients with increased relapse rates, increased sites of distant metastases and poor outcome [50]. In breast cancer, low OGA levels are linked to higher grade tumours and metastasis [51]. We have shown that the regulation of Uap1 by Fbx117 and an unidentified kinase, are factors in determining the levels of the $O$-GlcNAcylated proteome.

In conclusion, by surveying structural rearrangements in cancer databases, we discovered rearrangements commonly occur in FBXL17 which affect its ability to bind substrates and also assemble as part of a functional SCF ubiquitin ligase complex. By screening for Fbxl17 interacting proteins, we discovered Uap1 as a binding partner, but not a substrate of Fbxl17, and established that Fbxl17 is a negative regulator of global O-linked GlcNAcylation. The loss-offunction mutations in FBXL17 caused by structural rearrangements could have additional effects on the cell, since the targets of Fbxl17 are involved in major, cancer-relevant cellular processes.

\section{Materials and methods}

\section{Yeast two-hybrid assay}

The matchmaker gold yeast two-hybrid system (Clontech) was used to screen a human cDNA library (Mate \& Plate ${ }^{\mathrm{TM}}$ Library-Normalized, Universal Human (Clontech)). Fbxl17 aa 321 to 701 was PCR amplified and subcloned into pGBKT7 (Clontech). aa 321 to 586 was amplified from pGBKT7-FBXL17 plasmid and subcloned into pGBKT7 to create the $(\triangle 3 \mathrm{LRR})$ bait construct. 


\section{Purification of SCF ${ }^{\mathrm{Fbx} 117}$ complexes}

HEK293T cells were transfected with SCF components (Skp1, Cul1, Myc-Rbx1) and FLAG-Fbxl17 constructs. After $48 \mathrm{~h}$, cells were resuspended in lysis buffer (LB) (25 mM Tris-HCl, pH 7.5, $225 \mathrm{mM} \mathrm{KCl,} \mathrm{1 \%} \mathrm{NP-40)} \mathrm{with} \mathrm{a}$ protease inhibitor cocktail (Sigma-Aldrich) and phosphatase inhibitors (10 mM NaF, $1 \mathrm{mM}$ PMSF, $1 \mathrm{mM} \mathrm{Na} \mathrm{VO}_{4}$ ). Lysates were incubated with Anti-FLAG ${ }^{\circledR} \mathrm{M} 2$ Affinity Gel (Sigma-Aldrich) for $5 \mathrm{~h}$ at $4{ }^{\circ} \mathrm{C}$ with rotation. Beads were washed in LB and eluted in $300 \mu \mathrm{g} / \mathrm{mL}$ FLAG peptide (Sigma-Aldrich) in elution buffer (10 mM HEPES, pH 7.9, $225 \mathrm{mM} \mathrm{KCl}, 1.5 \mathrm{mM} \mathrm{MgCl}_{2}, 0.1 \% \mathrm{NP}-40$ ) for $1 \mathrm{~h}$ at $4{ }^{\circ} \mathrm{C}$ with rotation. Purified SCF complexes were stored in $15 \%$ glycerol.

\section{In vitro ubiquitination assays}

A screen of 10 different E2 enzymes determined that E2 UbcH5a enabled the most specific $\mathrm{SCF}^{\mathrm{Fbx} 117}$ activity and was used in subsequent experiments. Purified SCF complexes at 12.5, 25, 50 and $100 \mathrm{nM}$ were tested in the presence of a ubiquitin-mix (ubiquitin buffer, ubiquitin $(20 \mu \mathrm{M})$ E1 (UBE1, $100 \mathrm{nM}$ ), E2 (UbcH5a, $500 \mathrm{nM}$ ) and Mg-ATP (2 mM) (Boston Biochem)) incubated at $30{ }^{\circ} \mathrm{C}$ for $90 \mathrm{~min}$ to determine ligase activity by auto ubiquitination. $50 \mathrm{nM}$ of the SCF was sufficient for ligase activity and used in subsequent experiments. To test substrate ubiquitination substrates were transfected into HEK293T cells and immunoprecipitated using their indicated epitope tags conjugated to agarose beads. The purified substrate was then eluted from the beads and added as a component of the ubiquitin-mix. HA-Sufu was kindly provided by Vincenzo D'Angiolella (CRUK/MRC Oxford Institute for Radiation Oncology, Oxford, UK). Ubiquitination was detected by probing for the substrate or HA tag.

\section{In vivo ubiquitination assays}

HEK293T cells were transfected with expression constructs of interest, including myc-ubiquitin, and treated with $10 \mu \mathrm{M}$ MG132 (Sigma-Aldrich) $5 \mathrm{~h}$ prior to lysis. UAP1 was then immunoprecipitated with Monoclonal Anti-HA-Agarose antibody (Sigma-Aldrich). Modified UAP1 was detected with an endogenous UAP1 antibody. To test for phosphorylation $10 \mathrm{mM} \beta$-glycerophosphate or alkaline phosphatase (CIP) was added to the LB where indicated. LB containing CIP did not contain phosphatase inhibitors $\left(\mathrm{NaF}, \mathrm{Na}_{3} \mathrm{VO}_{4}\right)$.

\section{DNA constructs}

Coordinates and exon numbers for the FBXL17 gene are from Ensembl transcript ENST00000542267.5 (Fig. 1).
Human FBXL17 cDNA (GenScript) was subcloned into pcDNA3 and pcDNA3-FLAG. Truncation $(\triangle 3 \mathrm{LRR})$ and $(\triangle 10 \mathrm{LRR})$ and deletion constructs $(\Delta \mathrm{Fbox})$ were constructed by amplification or two-step PCR mutagenesis. Human Ufc1 and Uap1 cDNAs were obtained from GeneArt. pcDNA3.1-FLAG-hKLHL12 was kindly provided by S. Angers (University of Toronto, Canada). pCK2 V2N1_Venus2-HA-CSNK2B_N1 was kindly provided by A. Beck-Sickinger (Leipzig University, Germany).

\section{Antibodies}

The following antibodies were purchased anti- $\beta$-actin (Abcam, ab8227), anti-Cul1 (Santa Cruz, sc-11384), antiFbx117 (Genetex, GTX119211), anti-FLAG ${ }^{\circledR}$ M2 (SigmaAldrich, F3165), anti-Gal4 DBD (Santa Cruz, sc-510), anti-GAPDH (Sigma, G9545), anti-HA (Abcam, ab9110), anti-HA (C29F4) (Cell Signalling, 3724S), anti-Histone H1 (Santa Cruz, sc-8030), anti-Klhl12 (Abcam, ab14233), anti-myc tag (Cell Signalling, 2272), anti-O-GlcNAc (Covance, MMS-248R), anti-p19 (Skp1) (BD Biosciences, 610530), anti-UAP1 (Abcam, ab95949), anti-Ub (Santa Cruz, sc-8017), HRP-conjugated antibodies to mouse or rabbit IgG (Santa Cruz Biotechnology, sc-2055, sc-2313) or chicken IgY (Abcam, ab97135), Donkey anti-Rabbit and anti-Mouse IgG conjugated to Alexa Fluor 488 (Invitrogen). Signal detection was by enhanced chemiluminescence (ECL) (GE Healthcare).

\section{Cell culture, plasmids and transfection}

HB4a is an immortalised normal breast epithelial cell line from M.J. O'Hare [52]. Cell lines were maintained in DMEM supplemented with $10 \%$ foetal bovine serum (ThermoFisher), $2 \mathrm{mM}$ glutamine, $100 \mathrm{U} / \mathrm{mL}$ penicillin and streptomycin at $37{ }^{\circ} \mathrm{C}$ in a humidified $5 \% \mathrm{CO}_{2}$ atmosphere. Where indicated, cells were treated with $(50 \mu \mathrm{M})$ PugNAc, an inhibitor of $O$-GlcNAc- $\beta$ - $N$-acetylglucosaminidase (Oga), for $3 \mathrm{~h}$ prior to cell lysis.

\section{Immunoblotting and immunoprecipitation analysis}

Cells were lysed in protein extraction buffer $(20 \mathrm{mM}$ Tris-HCl pH 7.4, 1\% IGEPAL, $1 \%$ Triton X-100, $50 \mathrm{mM}$ $\mathrm{NaCl}, 2 \mathrm{mM}$ EDTA pH $8,30 \mathrm{mM} \mathrm{NaP} \mathrm{O}_{7}$ and protease inhibitor cocktail (Roche)). For the analysis of $O$-GlcNAcylated proteins, cells were lysed in RIPA buffer plus protease inhibitors.

For immunoprecipitation (IP) experiments, cells were lysed in hypotonic lysis buffer $(10 \mathrm{mM}$ Tris- $\mathrm{HCl} \mathrm{pH} 7.5$, $10 \mathrm{mM} \mathrm{NaCl}, 2 \mathrm{mM}$ EDTA, $0.5 \%$ Triton X-100 and protease 
inhibitors) and immunoprecipitated with agarose-anti-HA (Sigma) or agarose-anti-FLAG (Anti-FLAG ${ }^{\circledR}$ M2 Affinity Gel, Sigma-Aldrich) for $3.5 \mathrm{~h}$. Beads were pelleted and washed four times in $1 \times$ NET2 wash buffer $(50 \mathrm{mM}$ Tris- $\mathrm{HCl} \mathrm{pH} 7.5,150 \mathrm{mM} \mathrm{NaCl}, 0.05 \%$ Triton X-100). Bound proteins were eluted by addition of $40 \mu \mathrm{L} 2 \times$ Laemmli sample buffer and incubation at $60{ }^{\circ} \mathrm{C}$ for $3 \mathrm{~min}$.

\section{siRNA and shRNA expression}

siRNAs were purchased from Eurofins genomics and transfected at a final concentration of $\sim 60 \mathrm{nM}$ using Lipofectamine $^{\text {TM }}$ RNAiMAX (ThermoFisher). Sequences for the siRNAs were as follows:

\section{SiRNA2: GCAGAGAACTCAAAGATAT siRNA3: GGACAAACTCACTGATGAA}

$4 \times 10^{6} \psi \mathrm{Nx}$ cells were calcium phosphate transfected with shRNA or scRNA retroviral expression constructs with $25 \mu \mathrm{M}$ chloroquine (Sigma-Aldrich). 2 days post-transfection, $2 \times 10^{6}$ of target cells were infected with retroviruses in the presence of polybrene (Sigma-Aldrich).

Sequences for the shRNAs were as follows:

shRNA 1: GGACAAACTCACTGATGAAGG (targets exon 3 of $F B X L 17$ );

shRNA 2: GCTTGGACCTACGTCATATCA (targets exon 6 of $F B X L 17$ );

shRNA 3: AGGCATGATCGTCATAGCTAA (targets exon 4 of $F B X L 17)$.

The following day, cells were selected using $1.7 \mu \mathrm{g} / \mathrm{mL}$ of puromycin. After selection, RNA was extracted, reverse transcribed and quantified by qPCR. Expression level was normalised to GAPDH expression and was plotted relative to the expression of FBXL17 in the relevant control. Values represent mean \pm SEM of at least three independent experiments.

\section{Metabolites extraction and LC-MS analysis}

Cells were washed three times with PBS prior the extraction and $1 \mathrm{ml}$ of extraction buffer (50\% LC-MS grade methanol and $30 \%$ acetonitrile, $20 \%$ ultrapure water) was added per $1 \times 10^{6}$ cells. Cell were then incubated on dry ice for $15 \mathrm{~min}$, collected, kept under vigorous shaking for $15 \mathrm{~min}$ at $4{ }^{\circ} \mathrm{C}$, and left for $1 \mathrm{~h}$ incubation at $20^{\circ} \mathrm{C}$. Samples were centrifuged at $13,000 \mathrm{rpm}$ and supernatants were transferred to autosampler vials for LC-MS analysis. To avoid bias due to machine drift and processed blindly, samples were randomized. Q Exactive mass spectrometer (Thermo Fisher Scientific) coupled to a Dionex U3000 UHPLC (Thermo Fisher
Scientific) system was used to perform the LC-MS analysis. Sequant ZIC-pHILIC column (150mm $32.1 \mathrm{~mm})$ and guard column (20 mm $32.1 \mathrm{~mm}$ ) (Merck Millipore) were utilized for the chromatographic separation and the column oven temperature was maintained at $40{ }^{\circ} \mathrm{C}$. The mobile phase was composed of $20 \mathrm{mM}$ ammonium carbonate and $0.1 \%$ ammonium hydroxide in water (solvent $\mathrm{A}$ ), and acetonitrile (solvent B). The flow rate was set at $200 \mathrm{~mL} / \mathrm{min}$ with the gradient was programmed as follows: initially stayed at $20 \%$ of $\mathrm{A}$ and $80 \%$ of B for 2 min, then subjected to a linear increase to $80 \%$ of A and decrease to $20 \%$ of B in $15 \mathrm{~min}$. Both solvents were then brought back to initial condition and staid for $8 \mathrm{~min}$. The mass spectrometer was operated in full MS and tSIM (targeted Single Ion Monitoring), in positive and negative mode. XCalibur Qual Browser and XCalibur Quan Browser software (Thermo Fisher Scientific) were used to acquire the spectra and analyse the data.

\section{Quantitative PCR}

All qPCR reactions were performed in triplicates using LightCycler ${ }^{\circledR} 480$ SYBR Green Master Mix (Roche) according to manufacturer's instructions. The relative expression ratio of a target gene in comparison to a reference gene in a cDNA panel was quantified [53]. GAPDH was used as an endogenous housekeeping transcript. The relative expression level was based on the difference in $\mathrm{Ct}$ values between a control cell line such as HB4a and a sample cell line in the cDNA panel.

Acknowledgements Funding was provided by Breast Cancer Now (2013NovPhD172) to BM and HL and PAWE, and Cancer Research UK (C1023/A14545 and C1023/A9140) to PAWE; SF was supported by a Wildy Studentship from Gonville \& Caius College, and the Department of Pathology, University of Cambridge. FRT was funded by a BEPE-FAPESP fellowship (2012/09241-8). The Personalised Breast Cancer Program is funded by Addenbrookes Charitable Trust, the Mark Foundation and Cancer Research UK grants to CC and JEA. We thank the patients and staff of the Cambridge Breast Unit for providing and collecting tumours for sequencing. We also thank Christian Frezza, Efterpi Nikitopoulou and Laura Tronci for their LC-MS expertise.

Author contributions BM, SF, FRT, PAWE, and HL designed and conducted experiments, and interpreted results. RMG, OMR, JEA and CC provided and analysed genomics data. BM, PAWE and HL wrote the manuscript.

Open Access This article is distributed under the terms of the Creative Commons Attribution 4.0 International License (http://creativeco mmons.org/licenses/by/4.0/), which permits unrestricted use, distribution, and reproduction in any medium, provided you give appropriate credit to the original author(s) and the source, provide a link to the Creative Commons license, and indicate if changes were made. 


\section{References}

1. Edwards PA (2010) Fusion genes and chromosome translocations in the common epithelial cancers. J Pathol 220(2):244-254

2. Pao W, Girard N (2011) New driver mutations in non-small-cell lung cancer. Lancet Oncol 12(2):175-180

3. Bass AJ et al (2011) Genomic sequencing of colorectal adenocarcinomas identifies a recurrent VTI1A-TCF7L2 fusion. Nat Genet 43(10):964-968

4. Banerji S et al (2012) Sequence analysis of mutations and translocations across breast cancer subtypes. Nature 486(7403):405-409

5. Stephens PJ et al (2009) Complex landscapes of somatic rearrangement in human breast cancer genomes. Nature 462(7276):1005-1010

6. Nik-Zainal S et al (2012) Mutational processes molding the genomes of 21 breast cancers. Cell 149(5):979-993

7. Dulak AM et al (2013) Exome and whole-genome sequencing of esophageal adenocarcinoma identifies recurrent driver events and mutational complexity. Nat Genet 45(5):478-486

8. Baca SC et al (2013) Punctuated evolution of prostate cancer genomes. Cell 153(3):666-677

9. Curtis $\mathrm{C}$ et al (2012) The genomic and transcriptomic architecture of 2,000 breast tumours reveals novel subgroups. Nature 486(7403):346-352

10. Robinson DR et al (2011) Functionally recurrent rearrangements of the MAST kinase and Notch gene families in breast cancer. Nat Med 17(12):1646-1651

11. Skaar JR, Pagan JK, Pagano M (2013) Mechanisms and function of substrate recruitment by F-box proteins. Nat Rev Mol Cell Biol 14(6):369-381

12. Hershko A, Ciechanover A (1998) The ubiquitin system. Annu Rev Biochem 67:425-479

13. Wu S et al (2013) CAND1 controls in vivo dynamics of the cullin 1-RING ubiquitin ligase repertoire. Nat Commun 4:1642

14. Pierce NW et al (2013) Cand1 promotes assembly of new SCF complexes through dynamic exchange of $\mathrm{F}$ box proteins. Cell 153(1):206-215

15. Nelson DE, Randle SJ, Laman H (2013) Beyond ubiquitination: the atypical functions of Fbxo7 and other F-box proteins. Open Biol 3(10):130131

16. Lipkowitz S, Weissman AM (2011) RINGs of good and evil: RING finger ubiquitin ligases at the crossroads of tumour suppression and oncogenesis. Nat Rev Cancer 11(9):629-643

17. Randle SJ, Laman H (2016) F-box protein interactions with the hallmark pathways in cancer. Semin Cancer Biol 36:3-17

18. Gstaiger M et al (2001) Skp2 is oncogenic and overexpressed in human cancers. Proc Natl Acad Sci U S A 98(9):5043-5048

19. Frescas D, Pagano M (2008) Deregulated proteolysis by the F-box proteins SKP2 and beta-TrCP: tipping the scales of cancer. Nat Rev Cancer 8(6):438-449

20. Carrano AC et al (1999) SKP2 is required for ubiquitinmediated degradation of the CDK inhibitor p27. Nat Cell Biol 1(4):193-199

21. Nakayama K et al (2000) Targeted disruption of Skp2 results in accumulation of cyclin E and p27(Kip1), polyploidy and centrosome overduplication. EMBO J 19(9):2069-2081

22. Latres E et al (2001) Role of the F-box protein Skp2 in lymphomagenesis. Proc Natl Acad Sci USA 98(5):2515-2520

23. Lin HK et al (2010) Skp2 targeting suppresses tumorigenesis by Arf-p53-independent cellular senescence. Nature 464(7287):374-379

24. Akhoondi S et al (2007) FBXW7/hCDC4 is a general tumor suppressor in human cancer. Cancer Res 67(19):9006-9012

25. Bignell GR et al (2010) Signatures of mutation and selection in the cancer genome. Nature 463(7283):893-898
26. Schulte I et al (2012) Structural analysis of the genome of breast cancer cell line ZR-75-30 identifies twelve expressed fusion genes. BMC Genomics 13:719

27. Dawson SJ et al (2013) A new genome-driven integrated classification of breast cancer and its implications. EMBO J 32(5):617-628

28. Cerami E et al (2012) The cBio cancer genomics portal: an open platform for exploring multidimensional cancer genomics data. Cancer Discov 2(5):401-404

29. Raducu M et al (2016) SCF (Fbx117) ubiquitylation of Sufu regulates Hedgehog signaling and medulloblastoma development. EMBO J 35(13):1400-1416

30. Haltiwanger RS, Holt GD, Hart GW (1990) Enzymatic addition of O-GlcNAc to nuclear and cytoplasmic proteins. Identification of a uridine diphospho- $\mathrm{N}$-acetylglucosamine:peptide beta-Nacetylglucosaminyltransferase. J Biol Chem 265(5):2563-2568

31. Szumilo T et al (1996) Purification to homogeneity and properties of UDP-GlcNAc (GalNAc) pyrophosphorylase. J Biol Chem 271(22):13147-13154

32. Wang-Gillam A, Pastuszak I, Elbein AD (1998) A 17-amino acid insert changes UDP-N-acetylhexosamine pyrophosphorylase specificity from UDP-GalNAc to UDP-GlcNAc. J Biol Chem 273(42):27055-27057

33. Kreppel LK, Hart GW (1999) Regulation of a cytosolic and nuclear O-GlcNAc transferase. Role of the tetratricopeptide repeats. J Biol Chem 274(45):32015-32022

34. Itkonen HM et al (2015) UAP1 is overexpressed in prostate cancer and is protective against inhibitors of N-linked glycosylation. Oncogene 34(28):3744-3750

35. Davoli T et al (2013) Cumulative haploinsufficiency and triplosensitivity drive aneuploidy patterns and shape the cancer genome. Cell 155(4):948-962

36. Schulman BA et al (2000) Insights into SCF ubiquitin ligases from the structure of the Skp1-Skp2 complex. Nature 408(6810):381-386

37. Tan MK et al (2013) Parallel SCF adaptor capture proteomics reveals a role for SCFFBXL17 in NRF2 activation via BACH1 repressor turnover. Mol Cell 52(1):9-24

38. Mena EL et al (2018) Dimerization quality control ensures neuronal development and survival. Science 362:6411

39. Wagner SA et al (2011) A proteome-wide, quantitative survey of in vivo ubiquitylation sites reveals widespread regulatory roles. Mol Cell Proteomics 10(10):M111.013284

40. Gao S et al (2019) Reciprocal regulation between O-GlcNAcylation and $\beta$-catenin facilitates cell viability and inhibits apoptosis in liver cancer. DNA Cell Biol 38(4):286-296

41. Ong Q, Han W, Yang X (2018) O-GlcNAc as an integrator of signaling pathways. Front Endocrinol (Lausanne) 9:599

42. Yang X, Qian K (2017) Protein O-GlcNAcylation: emerging mechanisms and functions. Nat Rev Mol Cell Biol 18(7):452-465

43. Hart GW, Housley MP, Slawson C (2007) Cycling of O-linked beta- $\mathrm{N}$-acetylglucosamine on nucleocytoplasmic proteins. Nature 446(7139):1017-1022

44. Gambetta MC, Oktaba K, Müller J (2009) Essential role of the glycosyltransferase sxc/Ogt in polycomb repression. Science 325(5936):93-96

45. Netsirisawan $P$ et al (2015) Proteomic analysis reveals aberrant O-GlcNAcylation of extracellular proteins from breast cancer cell secretion. Cancer Genomics Proteomics 12(4):201-209

46. Ferrer CM et al (2014) O-GlcNAcylation regulates cancer metabolism and survival stress signaling via regulation of the HIF-1 pathway. Mol Cell 54(5):820-831 
47. Sodi VL et al (2015) mTOR/MYC axis regulates O-GlcNAc transferase expression and O-GlcNAcylation in breast cancer. Mol Cancer Res 13(5):923-933

48. Huang $X$ et al (2013) O-GlcNAcylation of cofilin promotes breast cancer cell invasion. J Biol Chem 288(51):36418-36425

49. Caldwell SA et al (2010) Nutrient sensor O-GlcNAc transferase regulates breast cancer tumorigenesis through targeting of the oncogenic transcription factor FoxM1. Oncogene 29(19):2831-2842

50. Tiainen S et al (2016) High extent of O-GlcNAcylation in breast cancer cells correlates with the levels of HAS enzymes, accumulation of hyaluronan, and poor outcome. Breast Cancer Res Treat 160(2):237-247

51. Krześlak A et al (2012) Gene expression of O-GlcNAc cycling enzymes in human breast cancers. Clin Exp Med 12(1):61-65
52. Stamps AC et al (1994) Analysis of proviral integration in human mammary epithelial cell lines immortalized by retroviral infection with a temperature-sensitive SV40 T-antigen construct. Int J Cancer 57(6):865-874

53. Pfaffl MW (2001) A new mathematical model for relative quantification in real-time RT-PCR. Nucleic Acids Res 29(9):e45

Publisher's Note Springer Nature remains neutral with regard to jurisdictional claims in published maps and institutional affiliations. 